\title{
铜催化加成/取代串联反应构建四取代的三吲哚甲烷
}

\author{
王文博韩华涁王乐乐 王琪琳* 卜站伟* \\ (河南大学化学化工学院 河南开封 475004)
}

\begin{abstract}
摘要 在温和的条件下, 利用 3-乙酰基吲哚与吲哚的加成/取代串联反应，高效地合成了一系列高度拥挤的四取代三吲 哚甲烷. 该方法通过在 3-乙酰基吲哚的氮原子上引入吸电子基团，提高了 3-乙酰基吲哚的反应活性，具有原料廉价易 得、底物适用性广、操作简便等优点.
\end{abstract}

关键词＼cjkstart吲哚; 三吲哚甲烷; 串联反应

\section{Access to Tetrasubstituted Tri(indolyl)methanes through Copper-Catalyzed Addition/Substitution Sequence}

\author{
Wang, Wenbo Han, Huabin Wang, Lele Wang, Qilin* Bu, Zhanwei* \\ (College of Chemistry and Chemical Engineering, Henan University, Kaifeng, Henan 475004)
}

\begin{abstract}
An efficient one-pot synthesis of highly crowded tetrasubstituted tri(indolyl)methanes has been achieved through the addition/substitution sequence of 3 -acetylindoles and indoles under mild conditions. The key to the success was improving the reactivity of 3 -acetylindoles by rationally installing the $\mathrm{N}$-protecting groups. The salient features including easily accessible and low-cost starting materials, board substrate scope, simple operation and structurally useful products make this approach particularly attractive.
\end{abstract}

Keywords indole; tri(indolyl)methane; cascade reaction

\section{Introduction}

Indoles are among the most important heterocyclic structural motifs that have been found in a plethora of natural products, functional materials and pharmaceuticals. ${ }^{[1-2]}$ Among them, tri-indolyl based compounds are quite significant due to their promising properties in terrestrial and marine natural products, and exhibit powerful bioactivities $^{[3]}$ (Figure 1). Specially, tri(indolyl)methanes, which are featured by three indole units connected through a simple methylene bridge, are very attractive not only because of their ubiquity in naturally occurring products but also because of their useful applications in synthetic chemistry. ${ }^{[4]}$ As a consequence, development of new and practical methods to access tri(indolyl)methanes is of great significance. To this end, some related synthetic methodologies have been well established, mainly involving the cascade reactions of indoles with active carbonyls or their surrogates, such as orthoformates,${ }^{[5]}$ acetic-formic anhydride, ${ }^{[6]}$
$N, N$-dimethylformaide dimethyl acetal, ${ }^{[7]} 3$-formylindole ${ }^{[8]}$ and in situ generated 3-formylindole from indolylglycine $^{[9]}$. Although these elegant cascade methodologies have the merits of operation simplification and step-economy, they often suffered from one or more intrinsic shortcomings, including the use of costly reagents, requirement of hazardous or corrosive promoters and limited substrate scope, etc.

Moreover, most of the above-mentioned methods focus on the construction of trisubstituted tri(indolyl)methanes, and the strategies for the preparation of much more crowded tetrasubstituted tri(indolyl)methanes have lagged far behind, ${ }^{[10]}$ which is not beneficial for structure-activity studies. Currently, it is still a quite challenging task to realize the direct construction of tetrasubstituted tri(indolyl)methanes because of the highly cumbersome architectures (Scheme 1, a). Recently, our group ${ }^{[11]}$ has successfully accomplished the synthesis of tetrasubstituted bis(indolyl)methanes through 1,2-addition/substitution sequential

\footnotetext{
* Corresponding authors. E-mail: wangqilin@henu.edu.cn; buzhanwei@henu.edu.cn

Received July 23, 2020; revised August 21, 2020; published online September 22, 2020.

Project supported by the National Natural Science Foundation of China (No. U1504206), the China Postdoctoral Science Foundation (No. 2020M672200) and the Graduate Education Innovation and Quality Improvement Program of Henan University (Nos. SYL19060137, SYL19030204).

国家自然科学基金(No. U1504206)、中国博士后科学基金(No. 2020M672200)和河南大学研究生教育创新与质量提升计划(Nos. SYL19060137, SYL19030204)资助项目.
} 


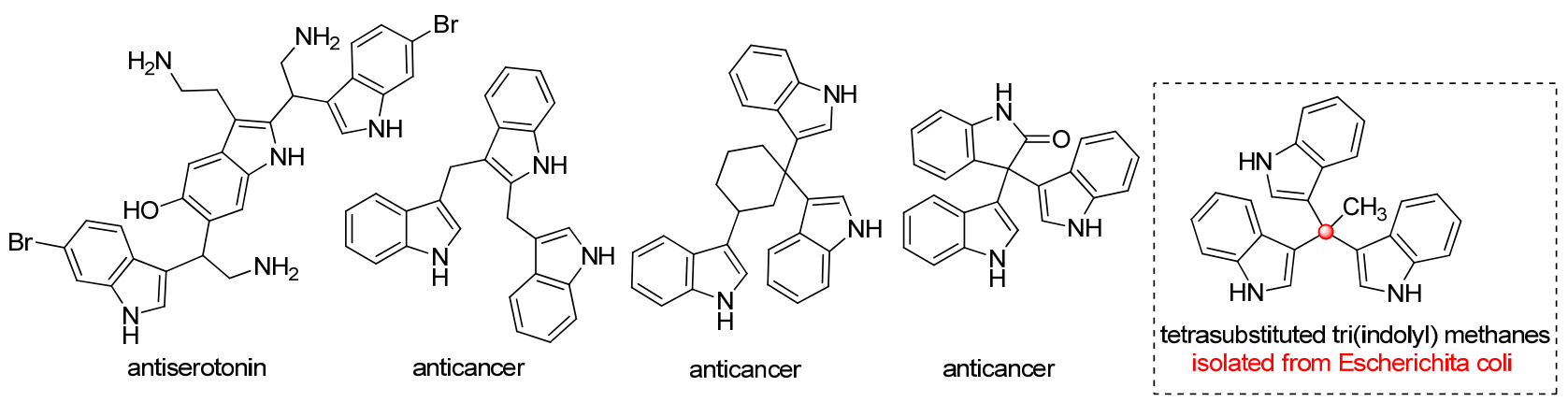

Figure 1 Selected triindoles derivatives with biological activities

(a) Previously reported tri(indolyl)methanes

Extensively studied<smiles>[R][R]1ccc2c(c1)c(Oc1cn([R])c3ccc([R])cc13)cn2[R]</smiles>

- Trisubstituted tri(indolyl)methanes

\section{Less studied}

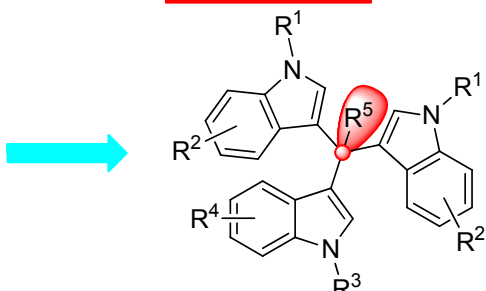

- Tetrasubstituted tri(indolyl)methanes

- more crowded (more challenging)

(b) Our previous work

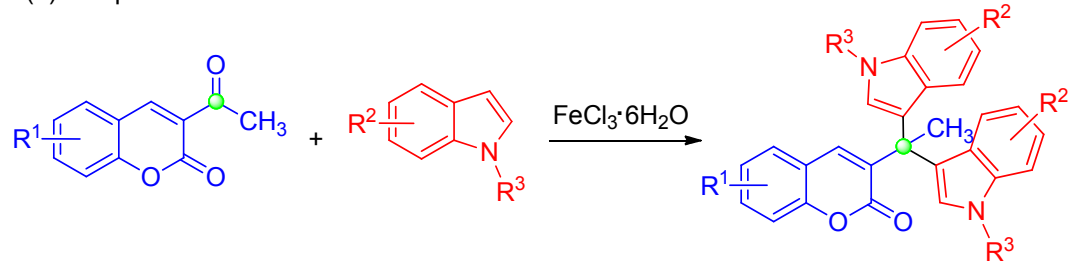

(c) This work<smiles>[R]n1ccc2cc[R1]([NH2+])cc21</smiles>
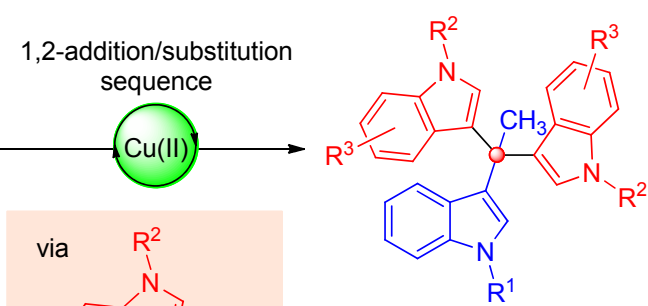

Synthetic challenges:

- less reactive 3-acetylindoles

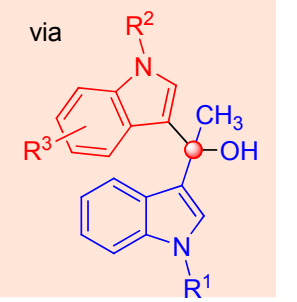

$\mathrm{R}^{1}$

o highly crowded tetrasubstituted tri(indolyl)methanes

Scheme 1 Our strategy for the construction of tetrasubstituted tri(indolyl)methanes based on addition/substitution sequence

strategy by employing coumarin-based ketones and indoles as reaction partners (Scheme 1, b). Inspired by this work, we envisioned that a similar reaction process may also occur among 3-acetylindole and two molecules of indoles under the catalysis of appropriate acid (Scheme 1, c). However, this seemingly facile reaction may be inhibited by the low reactivity of 3 -acetylindole, which was caused by the presence of electron-rich aromatic rings. Besides, after the first nucleophilic addition, the subsequent substitution may not proceed due to the unfavorable steric hindrance. As a continuation of our research interest in the construction of complex and diverse heterocycles, ${ }^{[12]}$ herein, we wish to report our preliminary results on the copper-catalyzed one-pot cascade reaction of 3-acetylindole and indoles for the synthesis of highly crowded tetrasubstituted tri(indolyl)methanes (Scheme 1, c). 


\section{Results and discussion}

To circumvent the low reactivity of 3-acetylindole, a series of electron-withdrawing groups were installed on the $\mathrm{N}$-atom. Initially, $\mathrm{N}$-tosyl substituted 3-acetylindole (1a) and $N$-benzyl indole (2a) were chosen as model substrates to test the feasibility of our synthetic strategy. Gratifyingly, the reaction proceeded smoothly in the presence of $\mathrm{FeCl}_{3} \cdot$ $6 \mathrm{H}_{2} \mathrm{O}$ at $35{ }^{\circ} \mathrm{C}$ in $\mathrm{MeCN}$ and went completion within 48 $\mathrm{h}$, furnishing the desired product $3 \mathrm{a}$ in $46 \%$ yield (Table 1, Entry 1). With this encouraging result, further optimizations were conducted to improve the yields. When $\mathrm{Fe}_{2}\left(\mathrm{SO}_{4}\right)_{3}$ was employed as the catalyst, the yield of 3a was enhanced to $82 \%$ (Table 1, Entry 2). Then, several easily available iron and copper salts and Brønsted acids were screened. Among them, $\mathrm{CuBr}_{2}$ turned out to be the most optimal, delivering 3a in 98\% yield (Table 1, Entry $5)$.

Table 1 Optimization of reaction conditions ${ }^{a}$

\begin{tabular}{|c|c|c|c|}
\hline 1a & $2 a$ & & $\mathrm{Bn}$ \\
\hline Entry & Cat. & Solvent & Yield $^{b} / \%$ \\
\hline 1 & $\mathrm{FeCl}_{3} \cdot 6 \mathrm{H}_{2} \mathrm{O}$ & $\mathrm{MeCN}$ & 46 \\
\hline 2 & $\mathrm{Fe}_{2}\left(\mathrm{SO}_{4}\right)_{3}$ & $\mathrm{MeCN}$ & 82 \\
\hline 3 & $\mathrm{Fe}(\mathrm{OTf})_{3}$ & $\mathrm{MeCN}$ & 72 \\
\hline 4 & $\mathrm{CuCl}_{2} \cdot 2 \mathrm{H}_{2} \mathrm{O}$ & $\mathrm{MeCN}$ & 77 \\
\hline 5 & $\mathrm{CuBr}_{2}$ & $\mathrm{MeCN}$ & 98 \\
\hline 6 & $\mathrm{Cu}(\mathrm{OTf})_{2}$ & $\mathrm{MeCN}$ & 82 \\
\hline 7 & $p$-TSA & $\mathrm{MeCN}$ & 95 \\
\hline 8 & TFA & $\mathrm{MeCN}$ & 一 \\
\hline 9 & $\mathrm{CuBr}_{2}$ & $\mathrm{CHCl}_{3}$ & 72 \\
\hline 10 & $\mathrm{CuBr}_{2}$ & THF & 34 \\
\hline 11 & $\mathrm{CuBr}_{2}$ & $\mathrm{MeOH}$ & 77 \\
\hline $12^{c}$ & $\mathrm{CuBr}_{2}$ & $\mathrm{MeCN}$ & $77^{d}$ \\
\hline $13^{e}$ & $\mathrm{CuBr}_{2}$ & $\mathrm{MeCN}$ & $75^{d}$ \\
\hline
\end{tabular}

${ }^{a}$ Unless otherwise specified, the reaction was conducted with $0.12 \mathrm{mmol}$ of $\mathbf{1 a}$ and $0.30 \mathrm{mmol}$ of $2 \mathrm{a}$ in $1 \mathrm{~mL}$ of solvent at $35{ }^{\circ} \mathrm{C} .{ }^{b}$ Isolated yield. ${ }^{c} 15 \mathrm{~mol} \%$ $\mathrm{CuBr}_{2} .{ }^{d} \mathrm{NMR}$ yield of the crude mixture using 1,3,5-trimethoxybenzene as the internal standard. ${ }^{e} 10 \mathrm{~mol} \% \mathrm{CuBr}_{2}$.

To further improve the synthetic efficiency, the effect of reaction media was evaluated (Table 1, Entries 9 11), and the results revealed that all gave inferior yields compared with $\mathrm{CH}_{3} \mathrm{CN}$. Lowering the loadings of $\mathrm{CuBr}_{2}$ to $15 \mathrm{~mol} \%$ and $10 \mathrm{~mol} \%$ was detrimental to the reaction with the formation of 3a in dramatically decreased yields (Table 1, Entries 12 and 13). Thus, the optimal condition for the formation of 3a was recommended to be $0.12 \mathrm{mmol}$ of $\mathbf{1 a}$, $0.30 \mathrm{mmol}$ of $2 \mathrm{a}$ with $20 \mathrm{~mol} \%$ of $\mathrm{CuBr}_{2}$ in $\mathrm{MeCN}$ at $35{ }^{\circ} \mathrm{C}$ for $48 \mathrm{~h}$.

With the optimal reaction conditions established, we turned our attention to examine the substrate scope (Table
2). First, the effect of $\mathrm{R}^{1}$ substituents on the $N$-atom of 3 -acetylindoles 1 was investigated, and the outcomes indicated that $\mathrm{R}^{1}$ could be anyone among Ts, Ns, Ac, Bz, Boc, $\mathrm{Cbz}$ and $\mathrm{CO}_{2}$ Et. They all participated in the coppercatalyzed cascade reactions successfully, delivering $\mathbf{3 a} \sim$ $3 \mathrm{~g}$ in moderate to good yields ( $41 \% \sim 98 \%$ yields, Table 2 , Entries $1 \sim 7$ ). It was noteworthy that $\mathrm{R}^{1}$ should be electron-withdrawing groups to guarantee sufficient reactivity. $\mathrm{N}$-Unsubstituted and $\mathrm{N}$-alkyl substituted 3-acetylindoles failed to participate in the reactions (not shown in Table 2). Subsequently, the generality of indoles was investigated. A wide range of $N$-alkyl groups were accommodated well irrespective of their bulkiness, allowing the construction of $\mathbf{3 h} \sim 3 \mathbf{m}$ in $57 \% \sim 88 \%$ yields. The reaction has good compatibility of $\mathrm{R}^{3}$ groups, regardless of their electronic properties and positions. Generally, the indoles with electron-donating groups exhibited much better reactivity, giving higher yields than the ones with electronwithdrawing groups (Table 2, Entries $14 \sim 16$ vs $17 \sim 21$ ). Remarkably, indoles bearing halogen groups on the aromatic rings were also compatible $(34 \% \sim 74 \%$ yields, Tab-

Table 2 Scope of substrates ${ }^{a}$

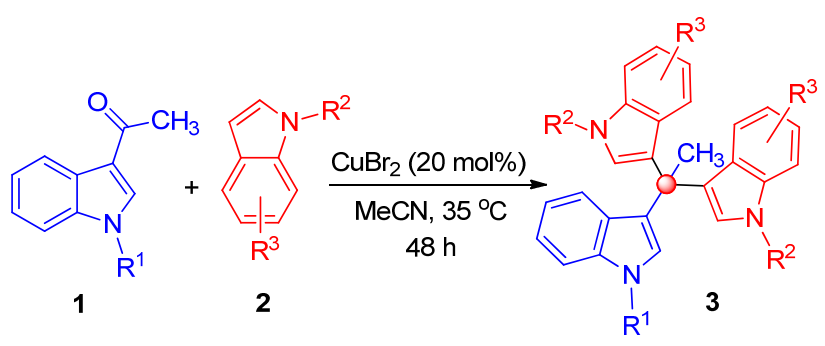

\begin{tabular}{cccccccc}
\hline Entry & $\mathrm{R}^{1}$ & $\mathbf{1}$ & $\mathrm{R}^{2}$ & $\mathrm{R}^{3}$ & $\mathbf{2}$ & $\mathbf{3}$ & $\mathrm{Yield}^{b} \%$ \\
\hline 1 & $\mathrm{Ts}$ & $\mathbf{1 a}$ & $\mathrm{Bn}$ & $\mathrm{H}$ & $\mathbf{2 a}$ & $\mathbf{3 a}$ & 98 \\
2 & $\mathrm{Ns}$ & $\mathbf{1 b}$ & $\mathrm{Bn}$ & $\mathrm{H}$ & $\mathbf{2 a}$ & $\mathbf{3 b}$ & 41 \\
3 & $\mathrm{Ac}$ & $\mathbf{1 c}$ & $\mathrm{Bn}$ & $\mathrm{H}$ & $\mathbf{2 a}$ & $\mathbf{3 c}$ & 52 \\
4 & $\mathrm{Bz}$ & $\mathbf{1 d}$ & $\mathrm{Bn}$ & $\mathrm{H}$ & $\mathbf{2 a}$ & $\mathbf{3 d}$ & 64 \\
5 & $\mathrm{Boc}$ & $\mathbf{1 e}$ & $\mathrm{Bn}$ & $\mathrm{H}$ & $\mathbf{2 a}$ & $\mathbf{3 e}$ & 44 \\
6 & $\mathrm{Cbz}$ & $\mathbf{1 f}$ & $\mathrm{Bn}$ & $\mathrm{H}$ & $\mathbf{2 a}$ & $\mathbf{3 f}$ & 63 \\
7 & $\mathrm{CO}_{2} \mathrm{Et}$ & $\mathbf{1 g}$ & $\mathrm{Bn}$ & $\mathrm{H}$ & $\mathbf{2 a}$ & $\mathbf{3 g}$ & 43 \\
8 & $\mathrm{Ts}$ & $\mathbf{1 a}$ & $\mathrm{Me}$ & $\mathrm{H}$ & $\mathbf{2 b}$ & $\mathbf{3 h}$ & 75 \\
9 & $\mathrm{Ts}$ & $\mathbf{1 a}$ & $\mathrm{Et}$ & $\mathrm{H}$ & $\mathbf{2 c}$ & $\mathbf{3 i}$ & 57 \\
10 & $\mathrm{Ts}$ & $\mathbf{1 a}$ & $n-\mathrm{Pr}$ & $\mathrm{H}$ & $\mathbf{2 d}$ & $\mathbf{3 j}$ & 79 \\
11 & $\mathrm{Ts}$ & $\mathbf{1 a}$ & $i-\mathrm{Pr}$ & $\mathrm{H}$ & $\mathbf{2 e}$ & $\mathbf{3 k}$ & 80 \\
12 & $\mathrm{Ts}$ & $\mathbf{1 a}$ & $n-\mathrm{Bu}$ & $\mathrm{H}$ & $\mathbf{2 f}$ & $\mathbf{3 l}$ & 88 \\
13 & $\mathrm{Ts}$ & $\mathbf{1 a}$ & $\mathrm{Allyl}$ & $\mathrm{H}$ & $\mathbf{2 g}$ & $\mathbf{3 m}$ & 68 \\
14 & $\mathrm{Ts}$ & $\mathbf{1 a}$ & $\mathrm{Bn}$ & $5-\mathrm{CH}_{3}$ & $\mathbf{2 h}$ & $\mathbf{3 n}$ & 70 \\
15 & $\mathrm{Ts}$ & $\mathbf{1 a}$ & $\mathrm{Bn}$ & $6-\mathrm{CH}_{3}$ & $\mathbf{2 i}$ & $\mathbf{3 o}$ & 64 \\
16 & $\mathrm{Ts}$ & $\mathbf{1 a}$ & $\mathrm{Bn}$ & $5-\mathrm{OBn}$ & $\mathbf{2 j}$ & $\mathbf{3 p}$ & 71 \\
17 & $\mathrm{Ts}$ & $\mathbf{1 a}$ & $\mathrm{Bn}$ & $5-\mathrm{Cl}$ & $\mathbf{2 k}$ & $\mathbf{3 q}$ & 34 \\
18 & $\mathrm{Ts}$ & $\mathbf{1 a}$ & $\mathrm{Bn}$ & $5-\mathrm{Br}$ & $\mathbf{2 l}$ & $\mathbf{3 r}$ & 69 \\
19 & $\mathrm{Ts}$ & $\mathbf{1 a}$ & $\mathrm{Bn}$ & $6-\mathrm{Br}$ & $\mathbf{2 m}$ & $\mathbf{3 s}$ & 74 \\
20 & $\mathrm{Ts}$ & $\mathbf{1 a}$ & $\mathrm{Bn}$ & $5-\mathrm{CO}_{2} \mathrm{Me}$ & $\mathbf{2 n}$ & $\mathbf{3 t}$ & 53 \\
21 & $\mathrm{Ts}$ & $\mathbf{1 a}$ & $\mathrm{Bn}$ & $5-\mathrm{NO}_{2}$ & $\mathbf{2 o}$ & $\mathbf{3 u}$ & 32 \\
\hline
\end{tabular}

${ }^{a}$ Unless otherwise specified, the reactions were conducted on a $0.12 \mathrm{mmol}$ scale of $\mathbf{1}$ with 2.5 equiv. of $\mathbf{2}$ with $20 \mathrm{~mol} \% \mathrm{CuBr}_{2}$ in $1 \mathrm{~mL}$ of $\mathrm{MeCN}$ at $35{ }^{\circ} \mathrm{C} .{ }^{b}$ Isolated yield. 
le 2, Entries 17 19), which offered valuable handles for the post-functionalization through transition metalcatalyzed couplings to enhance the structural diversity and complexity.

Inspired by the success of this cascade process, the substrate scope to other ketones was further expanded (Scheme 2). Both acetophenone (1h) and $N$-methyl isatin (1i) were well accommodated, thus allowing for the synthesis of $\mathbf{3 v}$ and $\mathbf{3 w}$ in $93 \%$ and $88 \%$ yields, respectively. Remarkably, chalcone (1j) could also participate in this cascade reaction successfully to access $\mathbf{3 x}$ bearing a useful alkenyl group in $31 \%$ yield with the simultaneous formation of the Michael adduct 4 in $47 \%$ yield.

To demonstrate the synthetic applicability of this methodology, a preparative-scale experiment was performed with $1.5 \mathrm{mmol}$ of $\mathbf{1 a}$ and 2.5 equiv. of $\mathbf{2 a}$ in the presence of catalytic amount of $\mathrm{CuBr}_{2}$ (Scheme 3). Gratefully, this reaction proceeded smoothly to give $\mathbf{3 a}$ in reduced but acceptable yield $(0.75 \mathrm{~g}, 71 \%$ yield $)$, thus indicating that the reaction could be scalable. Based on the experimental results and previous literature report, ${ }^{[13]}$ a plausible cascade reaction mechanism was proposed. As shown in Scheme 4, upon activation by copper(II), $N$-tosyl 3 -acetylindole (1a) was prone to undergo 1,2-addition with electron-rich indole $2 \mathbf{a}$ to generate intermediate II, which could be easily transformed into intermediate III with the help of $\mathrm{CuBr}_{2}$ as Lewis acid. Then, intermediate III was captured by another indole molecule to form the desired tetrasubstituted tri(indolyl)methane 3a. To shed some light on the reaction mechanism, HRMS analysis of the crude reaction mixture of $\mathbf{1 a}$ and $\mathbf{2} \mathbf{a}$ in the presence of $\mathrm{CuBr}_{2}$ was performed (Figure 2). And the signal peak of intermediate III could be detected after the reaction took place for $2 \mathrm{~h}$, which manifested that this reaction may proceed through an $S_{N} 1$ process. To further confirm this, 3-indolyl alcohol $\mathbf{5}$ was prepared. By subjecting $\mathbf{5}$ to the reaction system of $\mathbf{2 a}$ and catalytic amount of $\mathrm{CuBr}_{2}$ in $\mathrm{CH}_{3} \mathrm{CN}$, expectedly, the desired 3,3'-biindole oxindole 6 was afforded in 54\% yield, highlighting the formation of cation intermediate (Scheme $5)$.

\section{Conclusions}

In conclusion, the efficient assembly of highly hindered tetrasubstituted tri(indolyl)methanes through the copper-

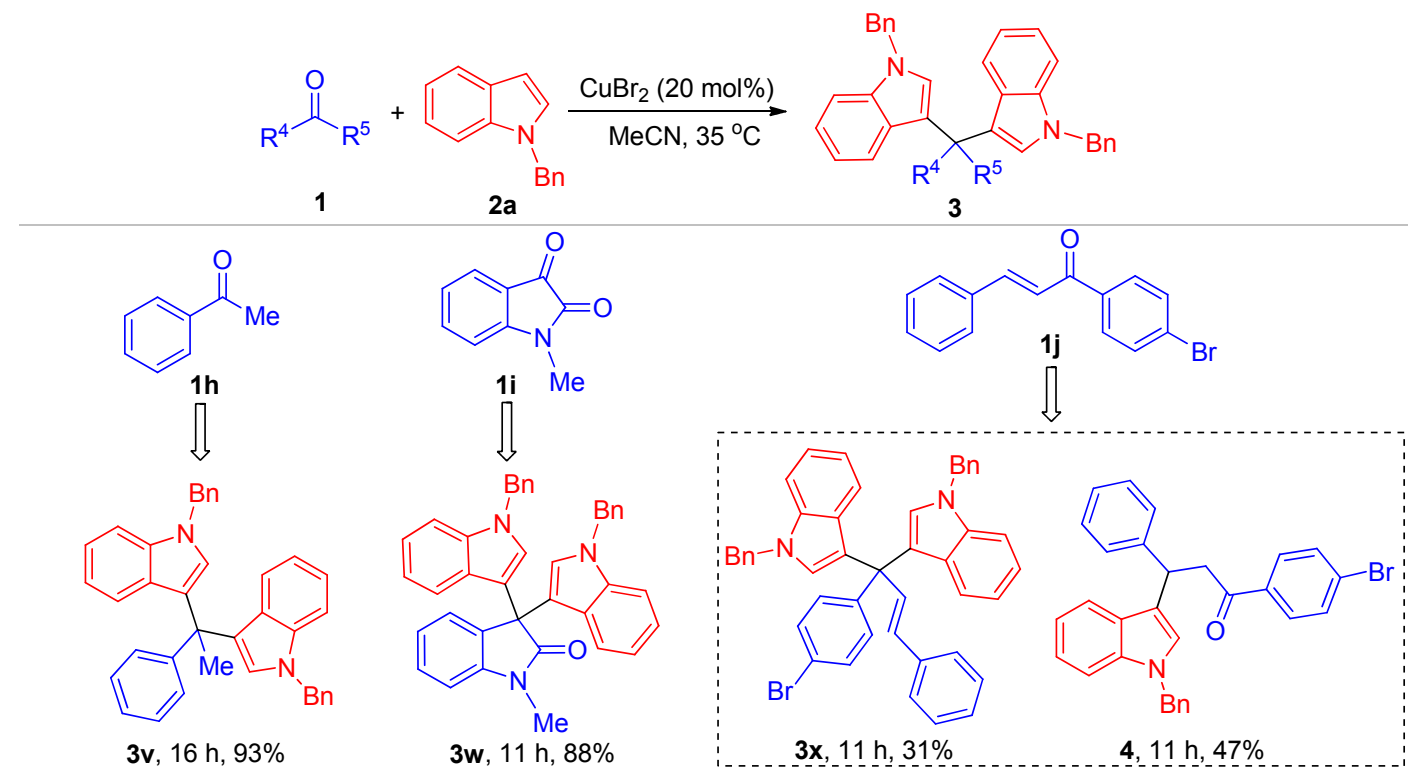

Scheme 2 Substrate scope with respect to different ketones

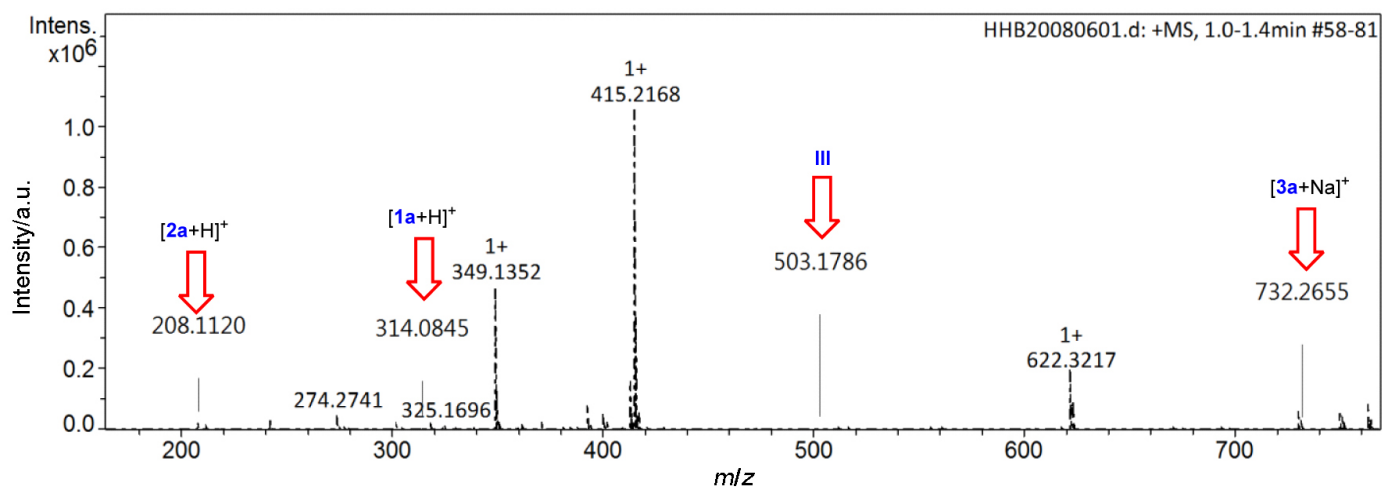

Figure 2 HRMS spectra of the crude reaction mixture between 1a and $2 \mathrm{a}$ for $2 \mathrm{~h}$ 


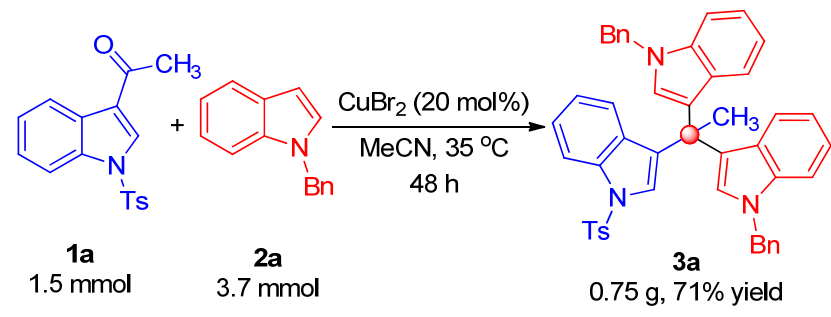

Scheme 3 Scalable preparation of $\mathbf{3 a}$

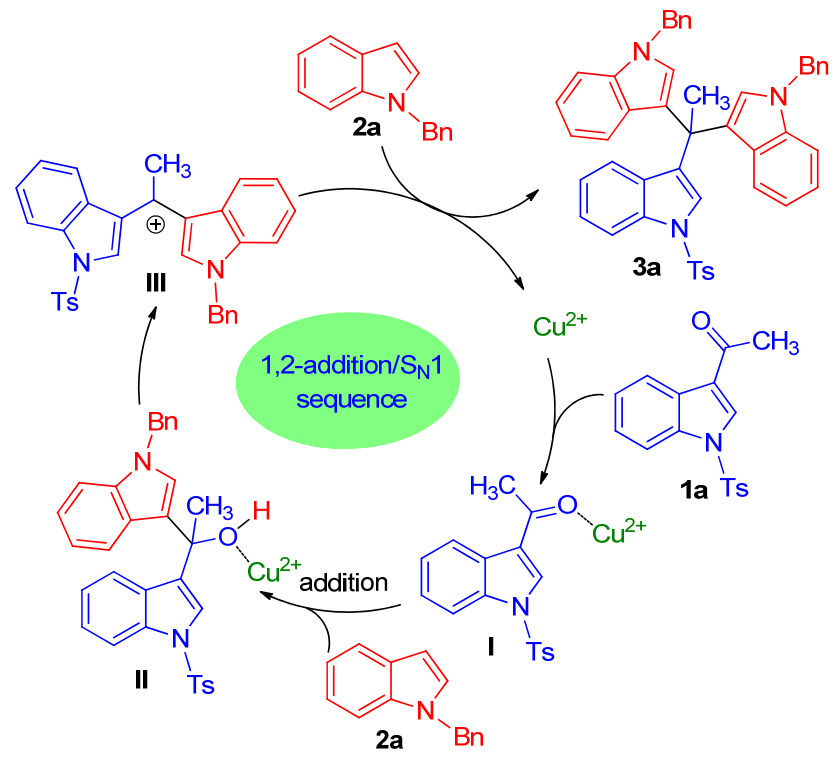

Scheme 4 Plausible mechanism

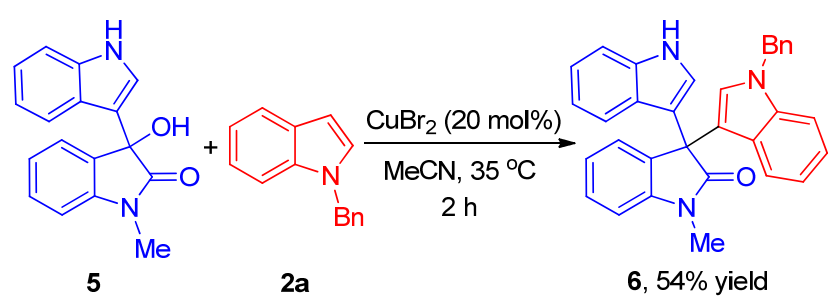

Scheme 5 Mechanistic elucidation using 3-indolyl alcohol as the substrate

catalyzed 1,2-addition/substitution sequence of 3-acetylindoles and indoles under mild conditions was successfully achieved. The key to the success was improving the reactivity of 3-acetylindoles by rationally tuning the $N$-protecting groups. The attractive advantages of this approach included easily accessible and low-cost starting materials, board substrate scope, mild conditions, simple operation and thus resultant structurally useful products. Further investigations to expand their applications in medicinal chemistry will be our focus.

\section{Experimental section}

\subsection{General methods}

NMR spectra were recorded with tetramethylsilane as the internal standard. ${ }^{1} \mathrm{H}$ NMR spectra were recorded at $400 \mathrm{MHz}$, and ${ }^{13} \mathrm{C} \mathrm{NMR}$ spectra were recorded at 100 $\mathrm{MHz}$ (Bruker Avance). ${ }^{1} \mathrm{H}$ NMR were reported relative to tetramethylsilane (TMS) with the solvent signal as the internal standard $\left(\mathrm{CDCl}_{3}\right.$ at $\left.\delta 7.26\right) .{ }^{13} \mathrm{C} \mathrm{NMR}$ were reported relative to tetramethylsilane (TMS) with the solvent resonance as the internal standard $\left(\mathrm{CDCl}_{3}\right.$ at $\left.\delta 77.00\right)$. Flash column chromatography was carried out using silica gel eluting with ethyl acetate and petroleum ether. High resolution mass spectra were obtained with a Q-TOF-Premier mass spectrometer. Reactions were monitored by thin-layer chromatography (TLC) and visualized with ultraviolet light. IR spectra were recorded on a Thermo Fisher Nicolet Avatar 360 FTIR spectrometer on $\mathrm{KBr}$ beam splitter. All solvents were used directly without any purification.

\subsection{General procedure for synthsis of tri(indolyl)- methanes 3}

To a $5.0 \mathrm{~mL}$ vial were successively added 3-acetylindoles 1 (0.12 mmol), indoles $2(0.30 \mathrm{mmol}), \mathrm{CuBr}_{2}(5.4$ $\mathrm{mg}, 0.024 \mathrm{mmol})$ and $1.0 \mathrm{~mL}$ of $\mathrm{CH}_{3} \mathrm{CN}$. The resulting mixture was stirred at $35{ }^{\circ} \mathrm{C}$ for $48 \mathrm{~h}$ until almost full consumption of 1 monitored by TLC, and then the reaction mixture was directly subjected to flash column chromatography on silica gel [ $V$ (petroleum ether) : $V$ (ethyl acetate) $=3: 1 \sim 5: 1]$ to afford the corresponding products.

3,3'-(1-(1-Tosyl-1H-indol-3-yl)ethane-1,1-diyl)bis(1benzyl-1H-indole) (3a): White solid, $83.4 \mathrm{mg}, 98 \%$ yield, reaction time 48 h. m.p. $121.5 \sim 122.7{ }^{\circ} \mathrm{C}$; ${ }^{1} \mathrm{H}$ NMR $(400$ $\left.\mathrm{MHz} \mathrm{CDCl}_{3}\right) \delta: 7.91(\mathrm{~d}, J=8.0 \mathrm{~Hz}, 1 \mathrm{H}), 7.61(\mathrm{t}, J=4.0$ $\mathrm{Hz}, 2 \mathrm{H}), 7.31 \sim 7.22(\mathrm{~m}, 4 \mathrm{H}), 7.14 \sim 7.07(\mathrm{~m}, 11 \mathrm{H}), 6.98$ $(\mathrm{t}, J=8.0 \mathrm{~Hz}, 2 \mathrm{H}), 6.88(\mathrm{~d}, J=4.0 \mathrm{~Hz}, 5 \mathrm{H}), 6.78(\mathrm{~d}, J=8.0$ $\mathrm{Hz}, 2 \mathrm{H}), 6.64(\mathrm{~s}, 2 \mathrm{H}), 5.08(\mathrm{~s}, 4 \mathrm{H}), 2.31(\mathrm{~s}, 3 \mathrm{H}), 2.22(\mathrm{~s}$, $3 \mathrm{H}) ;{ }^{13} \mathrm{C}$ NMR $\left(100 \mathrm{MHz}, \mathrm{CDCl}_{3}\right) \delta: 143.6,136.7,136.3$, $135.2,134.1,129.2,129.1,128.7,127.6,126.4,126.3$, $125.8,125.7,125.3,123.5,123.0,121.6,121.5,120.9$, $120.3,120.2,117.6,112.8,108.8,48.7,38.3,26.8,20.5$. IR (KBr) v: 3431, 3053, 2927, 1603, 1454, 1366, 1266, 1175, 1127, 1090, 1026, 806, 738, 692, $578 \mathrm{~cm}^{-1}$; HRMS (ESI) calcd for $\mathrm{C}_{47} \mathrm{H}_{39} \mathrm{~N}_{3} \mathrm{NaO}_{2} \mathrm{~S}[\mathrm{M}+\mathrm{Na}]^{+} 732.2655$, found 732.2644 .

3,3'-(1-(1-((4-Nitrophenyl)sulfonyl)-1H-indol-3-yl)ethane-1,1-diyl)bis(1-benzyl-1H-indole) (3b): White solid, $36.4 \mathrm{mg}, 41 \%$ yield, reaction time $=48$ h. m.p. $126.8 \sim$ $127.9{ }^{\circ} \mathrm{C} ;{ }^{1} \mathrm{H}$ NMR (400 MHz, $\left.\mathrm{CDCl}_{3}\right) \delta: 8.15$ (d, $J=8.0$ $\mathrm{Hz}, 2 \mathrm{H}), 7.91$ (d, $J=8.0 \mathrm{~Hz}, 1 \mathrm{H}), 7.86(\mathrm{~d}, J=8.0 \mathrm{~Hz}, 2 \mathrm{H})$, $7.30 \sim 7.15(\mathrm{~m}, 13 \mathrm{H}), 7.03(\mathrm{t}, J=8.0 \mathrm{~Hz}, 2 \mathrm{H}), 6.92(\mathrm{dd}$, $J=4.0,8.0 \mathrm{~Hz}, 5 \mathrm{H}), 6.81(\mathrm{t}, J=8.0 \mathrm{~Hz}, 2 \mathrm{H}), 6.67(\mathrm{~s}, 2 \mathrm{H})$, $5.14(\mathrm{~s}, 4 \mathrm{H}), 2.31(\mathrm{~s}, 3 \mathrm{H}) ;{ }^{13} \mathrm{C} \mathrm{NMR}\left(100 \mathrm{MHz}, \mathrm{CDCl}_{3}\right) \delta$ : $149.5,142.2,136.6,136.4,135.0,130.7,129.3,127.6$, $127.1,126.4,126.3,125.6,125.4,123.7,123.3,123.1$, $122.3,122.1,120.7,120.5,119.9,117.6,112.7,109.0$, $52.4,48.7,17.4$; IR (KBr) v: 3430, 3039, 2925, 2861, $1609,1532,1454,1378,1264,1179,1122,1025,805,737$, $689,617,572 \mathrm{~cm}^{-1}$; HRMS (ESI) calcd for $\mathrm{C}_{46} \mathrm{H}_{36} \mathrm{~N}_{4^{-}}$ $\mathrm{NaO}_{4} \mathrm{~S}[\mathrm{M}+\mathrm{Na}]^{+}$763.2349, found 763.2344.

1-(3-(1,1-Bis(1-benzyl-1H-indol-3-yl)ethyl)- $1 H$-indol-1yl)ethan-1-one (3c): White solid, $37.3 \mathrm{mg}, 52 \%$ yield, reaction time 48 h. m.p. $138.5 \sim 140.1{ }^{\circ} \mathrm{C} ;{ }^{1} \mathrm{H}$ NMR $(400$ $\left.\mathrm{MHz}, \mathrm{CDCl}_{3}\right) \delta: 7.42(\mathrm{~d}, J=8.0 \mathrm{~Hz}, 2 \mathrm{H}), 7.35$ (d, $J=8.0$ 
$\mathrm{Hz}, 1 \mathrm{H}), 7.16 \sim 7.13(\mathrm{~m}, 10 \mathrm{H}), 7.04 \sim 6.91(\mathrm{~m}, 8 \mathrm{H}), 6.85 \sim$ $6.79(\mathrm{~m}, 4 \mathrm{H}), 5.15(\mathrm{~s}, 4 \mathrm{H}), 2.38(\mathrm{~s}, 3 \mathrm{H}), 2.31(\mathrm{~s}, 3 \mathrm{H}) ;{ }^{13} \mathrm{C}$ NMR $\left(100 \mathrm{MHz}, \mathrm{CDCl}_{3}\right) \delta: 167.7,136.8,136.3,135.8$, $128.6,128.5,127.6,126.4,125.8,125.4,123.5,122.6$, $121.7,121.3,120.9,120.3,117.6,115.6,108.8$, two carbon missing in the aromatic region, 48.7, 38.3, 26.8, 23.4; IR (KBr) $v$ : 3431, 3038, 2928, 1702, 1608, 1454, 1380, 1330, $1255,1232,1178,1090,1025,804,738 \mathrm{~cm}^{-1}$; HRMS (ESI) calcd for $\mathrm{C}_{42} \mathrm{H}_{35} \mathrm{~N}_{3} \mathrm{NaO}[\mathrm{M}+\mathrm{Na}]^{+}$620.2672, found 620.2657.

(3-(1,1-Bis(1-benzyl-1H-indol-3-yl)ethyl)- $1 H$-indol-1yl)(phenyl)methanone (3d): White solid, $50.7 \mathrm{mg}, 64 \%$ yield, reaction time 48 h. m.p. $113.2 \sim 115.1{ }^{\circ} \mathrm{C} ;{ }^{1} \mathrm{H}$ NMR $\left(400 \mathrm{MHz}, \mathrm{CDCl}_{3}\right) \delta: 8.39(\mathrm{~d}, J=8.0 \mathrm{~Hz}, 1 \mathrm{H}), 7.47$ (q, $J=$ $8.0 \mathrm{~Hz}, 5 \mathrm{H}), 7.29(\mathrm{t}, J=8.0 \mathrm{~Hz}, 1 \mathrm{H}), 7.22(\mathrm{t}, J=8.0 \mathrm{~Hz}$, $1 \mathrm{H}), 7.10 \sim 6.99(\mathrm{~m}, 13 \mathrm{H}), 6.89 \sim 6.82(\mathrm{~m}, 7 \mathrm{H}), 6.75(\mathrm{~s}$, 2H), $5.10(\mathrm{~s}, 4 \mathrm{H}), 2.38(\mathrm{~s}, 3 \mathrm{H}) ;{ }^{13} \mathrm{C} \mathrm{NMR}(100 \mathrm{MHz}$, $\left.\mathrm{CDCl}_{3}\right) \delta: 168.5,137.9,137.5,137.3,134.3,131.7,130.0$, $129.5,129.1,128.7,128.2,127.4,126.9,126.6,126.4$, $124.5,123.2,122.5,122.1,121.5,121.4,118.7,116.6$, 110.0 , one carbon missing in the aromatic region, 49.7 , 39.4, 18.5; IR (KBr) v: 3434, 3055, 2924, 1682, 1454, $1365,1333,1257,1180,1147,1079,1023,872,801,740$, $705 \mathrm{~cm}^{-1}$; HRMS (ESI) calcd for $\mathrm{C}_{47} \mathrm{H}_{37} \mathrm{~N}_{3} \mathrm{NaO}[\mathrm{M}+\mathrm{Na}]^{+}$ 682.2829 , found 682.2807 .

tert-Butyl 3-(1,1-bis(1-benzyl-1H-indol-3-yl)ethyl)-1Hindole-1-carboxylate (3e): White solid, $34.6 \mathrm{mg}, 44 \%$ yield, reaction time 48 h. m.p. $102.7 \sim 104.1{ }^{\circ} \mathrm{C} ;{ }^{1} \mathrm{H}$ NMR $\left(400 \mathrm{MHz}, \mathrm{CDCl}_{3}\right) \delta: 7.47(\mathrm{~d}, J=8.0 \mathrm{~Hz}, 2 \mathrm{H}), 7.38$ (s, $1 \mathrm{H}), 7.29$ (d, $J=8.0 \mathrm{~Hz}, 1 \mathrm{H}), 7.14$ (dd, $J=4.0,12.0 \mathrm{~Hz}$, $10 \mathrm{H}), 6.99(\mathrm{t}, J=8.0 \mathrm{~Hz}, 2 \mathrm{H}), 6.91(\mathrm{~d}, J=8.0 \mathrm{~Hz}, 4 \mathrm{H})$, $6.84(\mathrm{dd}, J=8.0,4.0 \mathrm{~Hz}, 3 \mathrm{H}), 6.75(\mathrm{~s}, 2 \mathrm{H}), 5.14(\mathrm{~s}, 4 \mathrm{H})$, $2.38(\mathrm{~s}, 3 \mathrm{H}), 1.54(\mathrm{~s}, 9 \mathrm{H}) ;{ }^{13} \mathrm{C}$ NMR $\left(100 \mathrm{MHz}, \mathrm{CDCl}_{3}\right) \delta$ : $138.1,138.0,137.4$ (2C), 129.9, 128.6, 127.6, 127.3, $127.0,126.4,123.8,123.6,122.6,122.1,121.6,121.3$, $121.1,118.6,118.3,115.1,109.7,58.5,49.7,39.3,28.2$, 18.5; IR (KBr) v: 3430, 3053, 2972, 2927, 1730, 1456, 1370, 1257, 1158, 1101, 1060, 805, $739 \mathrm{~cm}^{-1}$; HRMS (ESI) calcd for $\mathrm{C}_{45} \mathrm{H}_{41} \mathrm{~N}_{3} \mathrm{NaO}_{2}[\mathrm{M}+\mathrm{Na}]^{+}$678.3091, found 678.3052 .

Benzyl 3-(1,1-bis(1-benzyl-1H-indol-3-yl)ethyl)- $1 H$-indole-1-carboxylate (3f): White solid, $52.1 \mathrm{mg}$, 63\% yield, reaction time 48 h. m.p. $82.5 \sim 83.7{ }^{\circ} \mathrm{C} ;{ }^{1} \mathrm{H}$ NMR $(400$ $\left.\mathrm{MHz}, \mathrm{CDCl}_{3}\right) \delta: 7.45(\mathrm{~d}, J=8.0 \mathrm{~Hz}, 2 \mathrm{H}), 7.40(\mathrm{~s}, 1 \mathrm{H})$, $7.31(\mathrm{~d}, J=8.0 \mathrm{~Hz}, 1 \mathrm{H}), 7.27(\mathrm{~s}, 5 \mathrm{H}), 7.16 \sim 7.10(\mathrm{~m}$, $10 \mathrm{H}), 7.00(\mathrm{t}, J=8.0 \mathrm{~Hz}, 2 \mathrm{H}), 6.92 \sim 6.87(\mathrm{~m}, 5 \mathrm{H}), 6.84(\mathrm{t}$, $J=8.0 \mathrm{~Hz}, 2 \mathrm{H}), 6.76(\mathrm{~s}, 2 \mathrm{H}), 5.31(\mathrm{~s}, 2 \mathrm{H}), 5.14(\mathrm{~s}, 4 \mathrm{H})$, $2.37(\mathrm{~s}, 3 \mathrm{H}) ;{ }^{13} \mathrm{C} \mathrm{NMR}\left(100 \mathrm{MHz}, \mathrm{CDCl}_{3}\right) \delta: 157.3,136.8$, $136.3,134.3,128.8,127.8,127.7,127.6,127.4,127.0$, $126.5,126.3,125.9,125.4,122.9,122.5,121.5,121.0$, $120.4,120.2,117.6,114.1,108.7$, two carbons missing in the aromatic region, 67.2, 48.7, 38.3, 17.4; IR (KBr) $v$ : 3432, 3039, 2961, 2923, 2856, 1734, 1612, 1455, 1390, 1251, 1179, 1095, 1057, 1027, 907, 805, $735 \mathrm{~cm}^{-1}$; HRMS (ESI) calcd for $\mathrm{C}_{48} \mathrm{H}_{39} \mathrm{~N}_{3} \mathrm{NaO}_{2}[\mathrm{M}+\mathrm{Na}]^{+}$712.2934, found 712.2913 .

Ethyl 3-(1,1-bis(1-benzyl-1H-indol-3-yl)ethyl)- $1 H$ - indole-1-carboxylate (3g): White solid, $32.4 \mathrm{mg}, 43 \%$ yield, reaction time 48 h. m.p. $73.7 \sim 74.6{ }^{\circ} \mathrm{C} ;{ }^{1} \mathrm{H}$ NMR $\left(400 \mathrm{MHz} \mathrm{CDCl}_{3}\right) \delta: 8.07(\mathrm{~d}, J=8.0 \mathrm{~Hz}, 1 \mathrm{H}), 7.45(\mathrm{~d}, J=$ $8.0 \mathrm{~Hz}, 2 \mathrm{H}), 7.40(\mathrm{~s}, 1 \mathrm{H}), 7.30(\mathrm{~d}, J=8.0 \mathrm{~Hz}, 1 \mathrm{H}), 7.18 \sim$ $7.11(\mathrm{~m}, 9 \mathrm{H}), 6.99(\mathrm{t}, J=8.0 \mathrm{~Hz}, 2 \mathrm{H}), 6.91(\mathrm{~d}, J=8.0 \mathrm{~Hz}$, $4 \mathrm{H}), 6.84(\mathrm{q}, J=8.0 \mathrm{~Hz}, 3 \mathrm{H}), 6.75(\mathrm{~s}, 2 \mathrm{H}), 5.13(\mathrm{~s}, 4 \mathrm{H})$, 4.35 (q, $J=8.0 \mathrm{~Hz}, 2 \mathrm{H}), 2.38(\mathrm{~s}, 3 \mathrm{H}), 1.33(\mathrm{t}, J=8.0 \mathrm{~Hz}$, $3 \mathrm{H}) ;{ }^{13} \mathrm{C}$ NMR $\left(100 \mathrm{MHz}, \mathrm{CDCl}_{3}\right) \delta: 137.9,137.4,129.9$, 128.7 (2C), 127.6, 127.4, 127.0, 126.4, 123.9, 123.3, $122.6,122.1,122.0,121.5,121.3,118.7,115.2,109.8$, 100.0 , one carbon missing in the aromatic region, 63.0, 49.8, 39.3, 28.1, 14.5; IR (KBr) v: 3437, 3043, 2975, 2926, 2864, 2249, 1733, 1610, 1546, 1455, 1384, 1248, 1177, 1096, 1062, 1021, 908, 805, $735 \mathrm{~cm}^{-1}$; HRMS (ESI) calcd for $\mathrm{C}_{43} \mathrm{H}_{37} \mathrm{~N}_{3} \mathrm{NaO}_{2}[\mathrm{M}+\mathrm{Na}]^{+} 650.2778$, found 650.2779 .

3,3'-(1-(1-Tosyl-1H-indol-3-yl)ethane-1,1-diyl)bis(1methyl-1H-indole) (3h): White solid, $50.2 \mathrm{mg}, 75 \%$ yield, reaction time 48 h. m.p. $178.7 \sim 180.2{ }^{\circ} \mathrm{C} ;{ }^{1} \mathrm{H}$ NMR $(400$ $\left.\mathrm{MHz}, \mathrm{CDCl}_{3}\right) \delta: 7.91(\mathrm{~d}, J=8.0 \mathrm{~Hz}, 1 \mathrm{H}), 7.61(\mathrm{~d}, J=8.0$ $\mathrm{Hz}, 2 \mathrm{H}), 7.25$ (t, $J=8.0 \mathrm{~Hz}, 3 \mathrm{H}), 7.16(\mathrm{q}, J=8.0 \mathrm{~Hz}, 5 \mathrm{H})$, $7.07(\mathrm{t}, J=8.0 \mathrm{~Hz}, 3 \mathrm{H}), 6.87 \sim 6.79(\mathrm{~m}, 3 \mathrm{H}), 6.47(\mathrm{~s}, 2 \mathrm{H})$, $3.53(\mathrm{~s}, 6 \mathrm{H}), 2.29(\mathrm{~s}, 6 \mathrm{H}) ;{ }^{13} \mathrm{C} \mathrm{NMR}\left(100 \mathrm{MHz}, \mathrm{CDCl}_{3}\right) \delta$ : $143.6,136.7,135.1,134.1,129.3,129.2,128.7,126.8$, $125.9,125.4,123.6,122.9,121.6,121.5,120.8,120.0$, $119.9,117.3,112.7,108.3,38.2,31.6,27.0,20.6$; IR (KBr) $v: 3435,3055,2931,1453,1368,1330,1173,1128,1093$, $1025,805,741,685,578 \mathrm{~cm}^{-1}$; HRMS (ESI) calcd for $\mathrm{C}_{35} \mathrm{H}_{31} \mathrm{~N}_{3} \mathrm{NaO}_{2} \mathrm{~S}[\mathrm{M}+\mathrm{Na}]^{+}$580.2029, found 580.2015.

3,3'-(1-(1-Tosyl-1H-indol-3-yl)ethane-1,1-diyl)bis(1ethyl-1H-indole) (3i): White solid, $40.1 \mathrm{mg}, 57 \%$ yield, reaction time 48 h. m.p. $199.1 \sim 200.9{ }^{\circ} \mathrm{C} ;{ }^{1} \mathrm{H}$ NMR $(400$ $\left.\mathrm{MHz}, \mathrm{CDCl}_{3}\right) \delta: 7.92(\mathrm{~d}, J=8.0 \mathrm{~Hz}, 1 \mathrm{H}), 7.63$ (d, $J=8.0$ $\mathrm{Hz}, 2 \mathrm{H}), 7.28(\mathrm{~d}, J=8.0 \mathrm{~Hz}, 2 \mathrm{H}), 7.23(\mathrm{~d}, J=8.0 \mathrm{~Hz}, 2 \mathrm{H})$, $7.19 \sim 7.16(\mathrm{~m}, 4 \mathrm{H}), 7.08(\mathrm{q}, J=8.0 \mathrm{~Hz}, 3 \mathrm{H}), 6.86(\mathrm{t}, J=$ $8.0 \mathrm{~Hz}, 1 \mathrm{H}), 6.81(\mathrm{t}, J=8.0 \mathrm{~Hz}, 2 \mathrm{H}), 6.55(\mathrm{~s}, 2 \mathrm{H}), 7.08(\mathrm{q}$, $J=8.0 \mathrm{~Hz}, 4 \mathrm{H}), 2.32(\mathrm{~s}, 3 \mathrm{H}), 2.29(\mathrm{~s}, 3 \mathrm{H}), 1.25(\mathrm{t}, J=8.0$ $\mathrm{Hz}, 6 \mathrm{H}) ;{ }^{13} \mathrm{C}$ NMR $\left(100 \mathrm{MHz}, \mathrm{CDCl}_{3}\right) \delta: 143.6,135.7$, $135.1,134.2,129.4,129.3,128.7,125.9,125.6,125.3$, $123.5,122.9,121.5$ (2C), 120.9, 119.8 (2C), 117.1, 112.7, 108.4, 39.7, 38.3, 26.9, 20.6, 14.6; IR (KBr) v: 3426, 3052, 2977, 2930, 1605, 1456, 1367, 1269, 1174, 1129, 1093, 1027, 808, 740, 684, $578 \mathrm{~cm}^{-1}$; HRMS (ESI) calcd for $\mathrm{C}_{37} \mathrm{H}_{35} \mathrm{~N}_{3} \mathrm{NaO}_{2} \mathrm{~S}[\mathrm{M}+\mathrm{Na}]^{+}$608.2342, found 608.2329.

3,3'-(1-(1-Tosyl-1H-indol-3-yl)ethane-1,1-diyl)bis(1propyl-1H-indole) (3j): White solid, $58.1 \mathrm{mg}, 79 \%$ yield, reaction time 48 h. m.p. 92.1 93.1 ${ }^{\circ} \mathrm{C} ;{ }^{1} \mathrm{H}$ NMR $(400$ $\left.\mathrm{MHz}, \mathrm{CDCl}_{3}\right) \delta: 7.91(\mathrm{~d}, J=8.0 \mathrm{~Hz}, 1 \mathrm{H}), 7.62$ (d, $J=8.0$ $\mathrm{Hz}, 2 \mathrm{H}), 7.27$ (d, $J=8.0 \mathrm{~Hz}, 2 \mathrm{H}), 7.25(\mathrm{~s}, 1 \mathrm{H}), 7.21 \sim 7.18$ $(\mathrm{m}, 3 \mathrm{H}), 7.14(\mathrm{~d}, J=8.0 \mathrm{~Hz}, 2 \mathrm{H}), 7.09 \sim 7.02(\mathrm{~m}, 3 \mathrm{H})$, $6.84(\mathrm{t}, J=8.0 \mathrm{~Hz}, 1 \mathrm{H}), 6.78(\mathrm{t}, J=8.0 \mathrm{~Hz}, 2 \mathrm{H}), 6.52(\mathrm{~s}$, $2 \mathrm{H}), 3.84(\mathrm{t}, J=8.0 \mathrm{~Hz}, 4 \mathrm{H}), 2.28(\mathrm{~s}, 6 \mathrm{H}), 1.68 \sim 1.60(\mathrm{~m}$, $4 \mathrm{H}), 0.73(\mathrm{t}, J=8.0 \mathrm{~Hz}, 6 \mathrm{H}) ;{ }^{13} \mathrm{C} \mathrm{NMR}\left(100 \mathrm{MHz}, \mathrm{CDCl}_{3}\right)$ $\delta: 143.6,136.0,135.1,134.2,129.4,129.3,128.7,126.1$, $125.9,125.6,123.5,122.9,121.6,121.4,120.9,119.8$, $119.4,117.1,112.7,108.5,46.7,38.3,26.9,22.4,20.6$, 10.4; IR (KBr) v: 3430, 2965, 2931, 2873, 1604, 1457, $1365,1175,1128,1094,1026,909,739,689,578 \mathrm{~cm}^{-1}$; 
HRMS (ESI) calcd for $\mathrm{C}_{39} \mathrm{H}_{39} \mathrm{~N}_{3} \mathrm{NaO}_{2} \mathrm{~S} \quad[\mathrm{M}+\mathrm{Na}]$ 636.2655, found 636.2627.

3,3'-(1-(1-Tosyl-1H-indol-3-yl)ethane-1,1-diyl)bis(1isopropyl-1H-indole) (3k): White solid, $58.9 \mathrm{mg}, 80 \%$ yield, reaction time 48 h. m.p. 97.6 $98.7{ }^{\circ} \mathrm{C}$; ${ }^{1} \mathrm{H}$ NMR $\left(400 \mathrm{MHz}, \mathrm{CDCl}_{3}\right) \delta: 7.92(\mathrm{~d}, J=8.0 \mathrm{~Hz}, 1 \mathrm{H}), 7.62(\mathrm{~d}, J=$ $8.0 \mathrm{~Hz}, 2 \mathrm{H}), 7.25(\mathrm{t}, J=8.0 \mathrm{~Hz}, 5 \mathrm{H}), 7.15(\mathrm{t}, J=8.0 \mathrm{~Hz}$, $3 \mathrm{H}), 7.09 \sim 7.01(\mathrm{~m}, 3 \mathrm{H}), 6.84(\mathrm{t}, J=8.0 \mathrm{~Hz}, 1 \mathrm{H}), 6.78(\mathrm{t}$, $J=8.0 \mathrm{~Hz}, 2 \mathrm{H}), 6.64(\mathrm{~s}, 2 \mathrm{H}), 4.52 \sim 4.46(\mathrm{~m}, 2 \mathrm{H}), 2.28(\mathrm{~s}$, $6 \mathrm{H}), 1.27(\mathrm{~d}, J=8.0 \mathrm{~Hz}, 12 \mathrm{H}) ;{ }^{13} \mathrm{C}$ NMR $(100 \mathrm{MHz}$, $\left.\mathrm{CDCl}_{3}\right) \delta: 144.7,136.7,136.3,135.3,130.6,130.4,129.8$, $127.0,126.6,124.6,124.0,123.0,122.7,122.5,122.0$, $120.8,120.7,118.2,113.9,109.6,46.8,39.6,28.0,22.7$, 21.7; IR (KBr) v: 3429, 3048, 2975, 2931, 1604, 1458, 1366, 1304, 1177, 1127, 1095, 1026, 805, 738, 686, 578 $\mathrm{cm}^{-1}$; HRMS (ESI) calcd for $\mathrm{C}_{39} \mathrm{H}_{39} \mathrm{~N}_{3} \mathrm{NaO}_{2} \mathrm{~S}[\mathrm{M}+\mathrm{Na}]^{+}$ 636.2655, found 636.2638.

3,3'-(1-(1-Tosyl-1H-indol-3-yl)ethane-1,1-diyl)bis(1butyl-1H-indole) (3l): White solid, $67.8 \mathrm{mg}, 88 \%$ yield, reaction time 48 h. m.p. $85.7 \sim 87.2{ }^{\circ} \mathrm{C} ;{ }^{1} \mathrm{H}$ NMR $(400$ $\left.\mathrm{MHz}, \mathrm{CDCl}_{3}\right) \delta: 7.92(\mathrm{~d}, J=8.0 \mathrm{~Hz}, 1 \mathrm{H}), 7.63(\mathrm{~d}, J=8.0$ $\mathrm{Hz}, 2 \mathrm{H}), 7.28 \sim 7.14(\mathrm{~m}, 8 \mathrm{H}), 7.10 \sim 7.03(\mathrm{~m}, 3 \mathrm{H}), 6.85(\mathrm{t}$, $J=8.0 \mathrm{~Hz}, 1 \mathrm{H}), 6.79(\mathrm{t}, J=8.0 \mathrm{~Hz}, 2 \mathrm{H}), 6.52(\mathrm{~s}, 2 \mathrm{H}), 3.89$ $(\mathrm{t}, J=8.0 \mathrm{~Hz}, 4 \mathrm{H}), 2.30(\mathrm{~s}, 3 \mathrm{H}), 2.29(\mathrm{~s}, 3 \mathrm{H}), 1.65 \sim 1.57$ $(\mathrm{m}, 4 \mathrm{H}), 1.17 \sim 1.09(\mathrm{~m}, 4 \mathrm{H}), 0.78(\mathrm{t}, J=8.0 \mathrm{~Hz}, 6 \mathrm{H}) ;{ }^{13} \mathrm{C}$ NMR $\left(100 \mathrm{MHz}, \mathrm{CDCl}_{3}\right) \delta: 143.6,136.0,135.1,134.2$, $129.4,129.2,128.7,126.1,125.9,125.5,123.5,122.9$, $121.6,121.4,120.9,119.8,119.4,117.1,112.7,108.5$, 44.8, 38.3, 31.2, 26.9, 20.6, 19.0, 12.7; IR (KBr) v: 3432, 3049, 2958, 2930, 2870, 1606, 1459, 1367, 1177, 1129, 1095, 1026, 807, $742 \mathrm{~cm}^{-1}$; HRMS (ESI) calcd for $\mathrm{C}_{41} \mathrm{H}_{43} \mathrm{~N}_{3} \mathrm{NaO}_{2} \mathrm{~S}[\mathrm{M}+\mathrm{Na}]^{+}$664.2968, found 664.2955.

3,3'-(1-(1-Tosyl-1H-indol-3-yl)ethane-1,1-diyl)bis(1allyl-1H-indole) (3m): White solid, $49.8 \mathrm{mg}, 68 \%$ yield, reaction time 48 h. m.p. $105.4 \sim 107.1{ }^{\circ} \mathrm{C} ;{ }^{1} \mathrm{H}$ NMR (400 $\left.\mathrm{MHz}, \mathrm{CDCl}_{3}\right) \delta: 7.91(\mathrm{~d}, J=8.0 \mathrm{~Hz}, 1 \mathrm{H}), 7.62(\mathrm{~d}, J=8.0$ $\mathrm{Hz}, 2 \mathrm{H}), 7.28$ (s, 1H), 7.25 (d, $J=4.0 \mathrm{~Hz}, 2 \mathrm{H}), 7.21 \sim 7.15$ $(\mathrm{m}, 5 \mathrm{H}), 7.11 \sim 7.03(\mathrm{~m}, 3 \mathrm{H}), 6.86(\mathrm{t}, J=8.0 \mathrm{~Hz}, 1 \mathrm{H}), 6.80$ $(\mathrm{t}, J=8.0 \mathrm{~Hz}, 2 \mathrm{H}), 6.55(\mathrm{~s}, 2 \mathrm{H}), 5.85 \sim 5.77(\mathrm{~m}, 2 \mathrm{H}), 5.03$ $(\mathrm{d}, J=12.0 \mathrm{~Hz}, 2 \mathrm{H}), 4.86(\mathrm{~d}, J=16.0 \mathrm{~Hz}, 2 \mathrm{H}), 4.51(\mathrm{~d}, J=$ $4.0 \mathrm{~Hz}, 4 \mathrm{H}), 2.31(\mathrm{~s}, 3 \mathrm{H}), 2.30(\mathrm{~s}, 3 \mathrm{H}) ;{ }^{13} \mathrm{C}$ NMR $(100$ $\left.\mathrm{MHz}, \mathrm{CDCl}_{3}\right) \delta: 143.8,136.0,135.0,133.8,129.2,128.6$, $128.1,127.9,127.5,127.3,126.6,125.8,125.3,123.4$, $122.8,121.7,121.5,119.4,111.3,110.6,57.4,49.1,38.1$, 26.8, 20.6, 17.4; IR (KBr) v: 3431, 3052, 2982, 2924, 1604, 1461, 1368, 1333, 1178, 1128, 1093, 1026, 807, 741, 693, $578 \mathrm{~cm}^{-1}$; HRMS (ESI) calcd for $\mathrm{C}_{39} \mathrm{H}_{35} \mathrm{~N}_{3} \mathrm{NaO}_{2} \mathrm{~S}$ $[\mathrm{M}+\mathrm{Na}]^{+}$632.2342, found 632.2335.

3,3'-(1-(1-Tosyl-1H-indol-3-yl)ethane-1,1-diyl)bis(1benzyl-5-methyl-1H-indole) (3n): White solid, $62.0 \mathrm{mg}$, $70 \%$ yield, reaction time 48 h. m.p. $119.7 \sim 121.4{ }^{\circ} \mathrm{C} ;{ }^{1} \mathrm{H}$ NMR (400 MHz, $\left.\mathrm{CDCl}_{3}\right) \delta: 7.87(\mathrm{~d}, J=8.0 \mathrm{~Hz}, 1 \mathrm{H}), 7.62$ $(\mathrm{d}, J=8.0 \mathrm{~Hz}, 2 \mathrm{H}), 7.29(\mathrm{t}, J=8.0 \mathrm{~Hz}, 2 \mathrm{H}), 7.17 \sim 7.06$ $(\mathrm{m}, 12 \mathrm{H}), 7.00(\mathrm{~d}, J=8.0 \mathrm{~Hz}, 2 \mathrm{H}), 6.89 \sim 6.80(\mathrm{~m}, 6 \mathrm{H})$, $6.59(\mathrm{~s}, 2 \mathrm{H}), 5.07(\mathrm{~s}, 4 \mathrm{H}), 2.34(\mathrm{~s}, 3 \mathrm{H}), 2.21(\mathrm{~s}, 9 \mathrm{H}) ;{ }^{13} \mathrm{C}$ NMR $\left(100 \mathrm{MHz}, \mathrm{CDCl}_{3}\right) \delta: 144.7,138.1,136.1,135.8$, $135.4,130.3,130.1,129.9,128.7,127.8,127.7,127.4$,
$127.1,126.8,126.4,124.4,124.0,123.0$ (2C), 122.5, $121.6,120.7,113.7,109.7,49.8,39.4,27.9,21.8,21.6$; IR (KBr) v: 3429, 3031, 2922, 2863, 1600, 1486, 1448, 1367, 1299, 1175, 1127, 1089, 1030, 786, 740, 696, $577 \mathrm{~cm}^{-1}$; HRMS (ESI) calcd for $\mathrm{C}_{49} \mathrm{H}_{43} \mathrm{~N}_{3} \mathrm{NaO}_{2} \mathrm{~S}[\mathrm{M}+\mathrm{Na}]^{+}$ 760.2968 , found 760.2993 .

3,3'-(1-(1-Tosyl-1H-indol-3-yl)ethane-1,1-diyl)bis(1benzyl-6-methyl-1H-indole) (3o): White solid, $57.1 \mathrm{mg}$, $64 \%$ yield, reaction time 48 h. m.p. $118.6 \sim 120.1{ }^{\circ} \mathrm{C} ;{ }^{1} \mathrm{H}$ NMR (400 MHz, $\left.\mathrm{CDCl}_{3}\right) \delta: 7.90(\mathrm{~d}, J=8.0 \mathrm{~Hz}, 1 \mathrm{H}), 7.62$ $(\mathrm{d}, J=8.0 \mathrm{~Hz}, 2 \mathrm{H}), 7.24 \sim 7.07(\mathrm{~m}, 13 \mathrm{H}), 6.90 \sim 6.83(\mathrm{~m}$, $7 \mathrm{H}), 6.62(\mathrm{~d}, J=8.0 \mathrm{~Hz}, 2 \mathrm{H}), 6.57(\mathrm{~s}, 2 \mathrm{H}), 5.08(\mathrm{~s}, 4 \mathrm{H})$, $2.28(\mathrm{t}, J=8.0 \mathrm{~Hz}, 12 \mathrm{H}) ;{ }^{13} \mathrm{C}$ NMR $\left(100 \mathrm{MHz}, \mathrm{CDCl}_{3}\right) \delta$ : $144.6,138.0,137.8,136.2,135.2,131.1,130.5,130.3$, $129.7,128.7,127.3,126.9,126.8,126.3,124.6$ (2C), $124.0,122.8,122.5,121.6,121.3,120.5,113.9,109.8$, 49.5, 39.3, 27.9, 21.8, 12.6; IR (KBr) v: 3428, 3028, 2922, 1610, 1544, 1454, 1369, 1174, 1124, 1030, 801, 740, 696, $578 \mathrm{~cm}^{-1}$; HRMS (ESI) calcd for $\mathrm{C}_{49} \mathrm{H}_{43} \mathrm{~N}_{3} \mathrm{NaO}_{2} \mathrm{~S}[\mathrm{M}+$ $\mathrm{Na}]^{+}$760.2968, found 760.2931 .

3,3'-(1-(1-Tosyl-1H-indol-3-yl)ethane-1,1-diyl)bis(1benzyl-5-(benzyloxy)-1H-indole) (3p): White solid, 78.6 mg, $71 \%$ yield, reaction time 48 h. m.p. $84.5 \sim 85.8{ }^{\circ} \mathrm{C} ;{ }^{1} \mathrm{H}$ NMR (400 MHz, $\left.\mathrm{CDCl}_{3}\right) \delta: 7.88(\mathrm{~d}, J=8.0 \mathrm{~Hz}, 1 \mathrm{H}), 7.59$ $(\mathrm{d}, J=8.0 \mathrm{~Hz}, 2 \mathrm{H}), 7.28 \sim 7.21(\mathrm{~m}, 11 \mathrm{H}), 7.17 \sim 7.08(\mathrm{~m}$, 9H), $7.03(\mathrm{~d}, J=8.0 \mathrm{~Hz}, 2 \mathrm{H}), 6.92(\mathrm{t}, J=8.0 \mathrm{~Hz}, 8 \mathrm{H}), 6.78$ $(\mathrm{d}, J=8.0 \mathrm{~Hz}, 2 \mathrm{H}), 6.65(\mathrm{~s}, 2 \mathrm{H}), 5.09(\mathrm{~s}, 4 \mathrm{H}), 4.73(\mathrm{~s}, 4 \mathrm{H})$, $2.29(\mathrm{~s}, 3 \mathrm{H}), 1.93(\mathrm{~s}, 3 \mathrm{H}) ;{ }^{13} \mathrm{C} \mathrm{NMR}\left(100 \mathrm{MHz}, \mathrm{CDCl}_{3}\right) \delta$ : $152.5,144.7,137.9,137.7,136.1,135.0,133.0,130.2$, $129.9,129.6,128.7,128.5,128.2,127.8,127.7,127.4$, $127.2,126.9,126.3,124.9,124.0,122.8,122.4,120.8$, $113.8,111.7,110.5,106.0,70.8,50.0,39.3,27.6,21.3$; IR (KBr) v: 3432, 3031, 2923, 1725, 1619, 1482, 1449, 1376, 1179, 1124, 1027, 910, 801, 741, 697, $579 \mathrm{~cm}^{-1}$; HRMS (ESI) calcd for $\mathrm{C}_{61} \mathrm{H}_{51} \mathrm{~N}_{3} \mathrm{NaO}_{4} \mathrm{~S}[\mathrm{M}+\mathrm{Na}]^{+}$944.3492, found 944.3518 .

3,3'-(1-(1-Tosyl-1H-indol-3-yl)ethane-1,1-diyl)bis(1benzyl-5-chloro- $1 H$-indole) (3q): White solid, $31.8 \mathrm{mg}$, $34 \%$ yield, reaction time 48 h. m.p. $120.3 \sim 122.0{ }^{\circ} \mathrm{C} ;{ }^{1} \mathrm{H}$ NMR (400 MHz, $\left.\mathrm{CDCl}_{3}\right) \delta: 7.90(\mathrm{~d}, J=8.0 \mathrm{~Hz}, 1 \mathrm{H}), 7.61$ $(\mathrm{d}, J=4.0 \mathrm{~Hz}, 1 \mathrm{H}), 7.49$ (d, $J=8.0 \mathrm{~Hz}, 1 \mathrm{H}), 7.29 \sim 6.90$ $(\mathrm{m}, 23 \mathrm{H}), 6.70(\mathrm{~s}, 1 \mathrm{H}), 5.11(\mathrm{~s}, 4 \mathrm{H}), 2.28(\mathrm{~s}, 3 \mathrm{H}), 2.21(\mathrm{~s}$, $3 \mathrm{H}) ;{ }^{13} \mathrm{C}$ NMR $\left(100 \mathrm{MHz}, \mathrm{CDCl}_{3}\right) \delta: 144.9,137.2,135.8$, $130.2,129.8,128.9$ (2C), 128.7, 127.7, 126.9, 126.3, $124.8,124.6,124.2,122.7,122.6,122.3,122.0,120.9$, $120.6,119.2,115.8,113.9,111.2,50.1,39.2,27.7,21.6$; IR (KBr) v: 3434, 3032, 2927, 1605, 1460, 1367, 1284, 1176, 1130, 1084, 1029, 799, 737, 693, $577 \mathrm{~cm}^{-1}$; HRMS (ESI) calcd for $\mathrm{C}_{47} \mathrm{H}_{37} \mathrm{Cl}_{2} \mathrm{~N}_{3} \mathrm{NaO}_{2} \mathrm{~S}[\mathrm{M}+\mathrm{Na}]^{+}$800.1876, found 800.1910 .

3,3'-(1-(1-Tosyl-1H-indol-3-yl)ethane-1,1-diyl)bis(1benzyl-5-bromo- $1 H$-indole) (3r): White solid, $71.8 \mathrm{mg}$, $69 \%$ yield, reaction time 48 h. m.p. $121.4 \sim 122.8{ }^{\circ} \mathrm{C} ;{ }^{1} \mathrm{H}$ NMR (400 MHz, $\left.\mathrm{CDCl}_{3}\right) \delta: 7.89(\mathrm{~d}, J=8.0 \mathrm{~Hz}, 1 \mathrm{H}), 7.63$ $(\mathrm{d}, J=8.0 \mathrm{~Hz}, 2 \mathrm{H}), 7.47$ (s, 2H), $7.30(\mathrm{~d}, J=8.0 \mathrm{~Hz}, 1 \mathrm{H})$, $7.25 \sim 7.14(\mathrm{~m}, 10 \mathrm{H}), 7.10(\mathrm{~d}, J=12.0 \mathrm{~Hz}, 2 \mathrm{H}), 7.00$ (d, $J=4.0 \mathrm{~Hz}, 2 \mathrm{H}), 6.92(\mathrm{~d}, J=4.0 \mathrm{~Hz}, 5 \mathrm{H}), 6.69$ (s, 2H), 5.13 
$(\mathrm{s}, 4 \mathrm{H}), 2.30(\mathrm{~s}, 3 \mathrm{H}), 2.24(\mathrm{~s}, 3 \mathrm{H}) ;{ }^{13} \mathrm{C}$ NMR $(100 \mathrm{MHz}$, $\left.\mathrm{CDCl}_{3}\right) \delta: 144.7,137.2,136.2,135.3,133.6,130.3,129.8$, $127.1,127.0,126.7,124.6,124.0,122.6,122.5,122.0$, $121.2,121.1,118.5,116.7,113.8,109.9$, three carbons missing in the aromatic region, 48.6, 39.4, 28.0, 21.7; IR (KBr) v: 3430, 2962, 2924, 1602, 1542, 1463, 1367, 1264, 1176, 1129, 1091, 1027, 803, 740, 693, $577 \mathrm{~cm}^{-1}$; HRMS (ESI) calcd for $\mathrm{C}_{47} \mathrm{H}_{37} \mathrm{Br}_{2} \mathrm{~N}_{3} \mathrm{NaO}_{2} \mathrm{~S}[\mathrm{M}+\mathrm{Na}]^{+}$888.0865, found 888.0870 .

3,3'-(1-(1-Tosyl-1H-indol-3-yl)ethane-1,1-diyl)bis(1benzyl-6-bromo- $1 H$-indole) (3s): White solid, $77.4 \mathrm{mg}$, $74 \%$ yield, reaction time 48 h. m.p. $122.8 \sim 124.4{ }^{\circ} \mathrm{C} ;{ }^{1} \mathrm{H}$ NMR (400 MHz, $\left.\mathrm{CDCl}_{3}\right) \delta: 7.94(\mathrm{~d}, J=8.0 \mathrm{~Hz}, 1 \mathrm{H}), 7.60$ $(\mathrm{d}, J=8.0 \mathrm{~Hz}, 2 \mathrm{H}), 7.29(\mathrm{~s}, 2 \mathrm{H}), 7.23 \sim 7.16(\mathrm{~m}, 9 \mathrm{H}), 7.12$ $(\mathrm{d}, J=8.0 \mathrm{~Hz}, 2 \mathrm{H}), 7.06(\mathrm{~d}, J=12.0 \mathrm{~Hz}, 2 \mathrm{H}), 6.91 \sim 6.83$ $(\mathrm{m}, 7 \mathrm{H}), 6.61(\mathrm{~s}, 2 \mathrm{H}), 5.09(\mathrm{~s}, 4 \mathrm{H}), 2.30(\mathrm{~s}, 3 \mathrm{H}), 2.24(\mathrm{~s}$, $3 \mathrm{H}) ;{ }^{13} \mathrm{C}$ NMR $\left(100 \mathrm{MHz}, \mathrm{CDCl}_{3}\right) \delta: 144.9,138.2,137.1$, $136.2,135.2,129.8$ (2C), 129.7, 128.8, 127.8, 127.7, $126.8,126.3,125.4,124.5,124.3,122.8,122.7,122.4$, $122.1,121.4,115.4,114.0,112.9,49.8,39.1,27.8,21.6$; IR (KBr) v: 3426, 2923, 2856, 1602, 1454, 1377, 1175, 1090, 1027, 806, 737, 695, $578 \mathrm{~cm}^{-1}$; HRMS (ESI) calcd for $\mathrm{C}_{47} \mathrm{H}_{37} \mathrm{Br}_{2} \mathrm{~N}_{3} \mathrm{NaO}_{2} \mathrm{~S}[\mathrm{M}+\mathrm{Na}]^{+}$888.0865, found 888.0831.

Dimethyl 3,3'-(1-(1-tosyl-1H-indol-3-yl)ethane-1,1-diyl)bis(1-benzyl-1H-indole-5-carboxylate) (3t): White solid, $52.5 \mathrm{mg}, 53 \%$ yield, reaction time 48 h. m.p. 128.5 $130.1{ }^{\circ} \mathrm{C} ;{ }^{1} \mathrm{H}$ NMR (400 MHz, $\mathrm{CDCl}_{3}$ ) $\delta: 8.19$ (s, 2H), $7.82(\mathrm{~d}, J=8.0 \mathrm{~Hz}, 1 \mathrm{H}), 7.74(\mathrm{~d}, J=8.0 \mathrm{~Hz}, 2 \mathrm{H}), 7.58$ (d, $J=8.0 \mathrm{~Hz}, 2 \mathrm{H}), 7.28(\mathrm{~d}, J=8.0 \mathrm{~Hz}, 1 \mathrm{H}), 7.17 \sim 7.09(\mathrm{~m}$, $11 \mathrm{H}), 6.92(\mathrm{~s}, 6 \mathrm{H}), 6.71(\mathrm{~s}, 2 \mathrm{H}), 5.15(\mathrm{~s}, 4 \mathrm{H}), 3.76(\mathrm{~s}, 6 \mathrm{H})$, $2.44(\mathrm{~s}, 3 \mathrm{H}), 2.24(\mathrm{~s}, 3 \mathrm{H}) ;{ }^{13} \mathrm{C} \mathrm{NMR}\left(100 \mathrm{MHz}, \mathrm{CDCl}_{3}\right) \delta$ : $166.9,143.6,138.9,135.9,134.8,134.0,129.0,128.6$, $128.0,127.9,127.8,126.6,125.9,125.4,125.2,123.7$, $123.5,123.1,122.0,121.7,121.5,121.4,119.9,112.6$, 108.8, 52.4, 50.7, 49.1, 38.4, 20.6; IR (KBr) v: 3431, 2923, 1710, 1611, 1441, 1379, 1252, 1177, 1129, 1091, 807, 740, $579 \mathrm{~cm}^{-1}$; HRMS (ESI) calcd for $\mathrm{C}_{51} \mathrm{H}_{43} \mathrm{~N}_{3} \mathrm{NaO}_{6} \mathrm{~S}[\mathrm{M}+$ $\mathrm{Na}]^{+}$848.2765, found 848.2797.

3,3'-(1-(1-Tosyl-1H-indol-3-yl)ethane-1,1-diyl)bis(1benzyl-5-nitro- $1 H$-indole) (3u): White solid, $30.7 \mathrm{mg}, 32 \%$ yield, reaction time 48 h. m.p. $138.6 \sim 139.7{ }^{\circ} \mathrm{C} ;{ }^{1} \mathrm{H}$ NMR $\left(400 \mathrm{MHz}, \mathrm{CDCl}_{3}\right) \delta: 8.23(\mathrm{~s}, 2 \mathrm{H}), 7.90(\mathrm{t}, J=8.0 \mathrm{~Hz}, 3 \mathrm{H})$, $7.57(\mathrm{~d}, J=8.0 \mathrm{~Hz}, 2 \mathrm{H}), 7.32(\mathrm{~d}, J=8.0 \mathrm{~Hz}, 1 \mathrm{H}), 7.24 \sim$ $7.14(\mathrm{~m}, 12 \mathrm{H}), 7.00 \sim 6.92(\mathrm{~m}, 7 \mathrm{H}), 5.20(\mathrm{~s}, 4 \mathrm{H}), 2.39(\mathrm{~s}$, $3 \mathrm{H}), 2.24(\mathrm{~s}, 3 \mathrm{H}) ;{ }^{13} \mathrm{C} \mathrm{NMR}\left(100 \mathrm{MHz}, \mathrm{CDCl}_{3}\right) \delta: 144.3$, $140.1,139.2,134.9$ (2C), 133.6, 129.3, 129.1, 128.1 (2C), $127.3,127.1,125.7,125.4,124.7,123.6,123.5,122.2$, $121.6,121.5,117.2,116.5,112.9,109.2,49.5,38.2,27.1$, 20.6; IR (KBr) v: 3432, 3070, 2964, 1613, 1516, 1371, 1332, 1263, 1176, 1131, 1080, 1029, 807,737, 694, 578 $\mathrm{cm}^{-1}$; HRMS (ESI) calcd for $\mathrm{C}_{47} \mathrm{H}_{37} \mathrm{~N}_{5} \mathrm{NaO}_{6} \mathrm{~S}[\mathrm{M}+\mathrm{Na}]^{+}$ 822.2357 , found 822.2360 .

3,3'-(1-Phenylethane-1,1-diyl)bis(1-benzyl-1 $H$-indole) (3v): White solid, $57.4 \mathrm{mg}, 93 \%$ yield, reaction time $16 \mathrm{~h}$. m.p. 57.8 58.3 ${ }^{\circ} \mathrm{C} ;{ }^{1} \mathrm{H}$ NMR $\left(400 \mathrm{MHz}, \mathrm{CDCl}_{3}\right) \delta: 7.43$ $(\mathrm{d}, J=8.0 \mathrm{~Hz}, 2 \mathrm{H}), 7.31(\mathrm{~d}, J=8.0 \mathrm{~Hz}, 2 \mathrm{H}), 7.25 \sim 7.16$ $(\mathrm{m}, 11 \mathrm{H}), 7.06(\mathrm{~d}, J=8.0 \mathrm{~Hz}, 2 \mathrm{H}), 7.01(\mathrm{~d}, J=8.0 \mathrm{~Hz}$,
$4 \mathrm{H}), 6.87(\mathrm{t}, J=8.0 \mathrm{~Hz}, 2 \mathrm{H}), 6.67(\mathrm{~s}, 2 \mathrm{H}), 5.18(\mathrm{~s}, 4 \mathrm{H})$, $2.36(\mathrm{~s}, 3 \mathrm{H}) ;{ }^{13} \mathrm{C}$ NMR $\left(100 \mathrm{MHz}, \mathrm{CDCl}_{3}\right) \delta: 148.0,137.8$, $137.4,128.6,128.1,127.8,127.6,127.3,127.2,126.4$, $125.8,123.7,122.2,121.2,118.6,109.7,49.8,43.8,29.3$. IR $(\mathrm{KBr}) v: 3453,1636,1562,732 \mathrm{~cm}^{-1}$; HRMS (ESI) calcd for $\mathrm{C}_{38} \mathrm{H}_{33} \mathrm{~N}_{2}[\mathrm{M}+\mathrm{H}]^{+}$517.2638, found 517.2632.

1,1"-Dibenzyl-1'-methyl-1H,1" $H$-[3,3':3',3"-terindol]$2^{\prime}\left(1^{\prime} H\right)$-one $(3 \mathbf{w})$ : White solid, $58.6 \mathrm{mg}, 88 \%$ yield, reaction time 11 h. m.p. $232.7 \sim 233.1{ }^{\circ} \mathrm{C} ;{ }^{1} \mathrm{H}$ NMR $(400$ $\left.\mathrm{MHz} \mathrm{CDCl}_{3}\right) \delta: 7.44(\mathrm{~d}, J=8.0 \mathrm{~Hz}, 1 \mathrm{H}), 7.34(\mathrm{~d}, J=8.0$ $\mathrm{Hz}, 2 \mathrm{H}), 7.32 \sim 7.20(\mathrm{~m}, 7 \mathrm{H}), 7.16(\mathrm{~d}, J=8.0 \mathrm{~Hz}, 2 \mathrm{H})$, $7.07 \sim 6.95(\mathrm{~m}, 10 \mathrm{H}), 6.90(\mathrm{t}, J=8.0 \mathrm{~Hz}, 2 \mathrm{H}), 5.20(\mathrm{~s}, 4 \mathrm{H})$, $3.33(\mathrm{~s}, 3 \mathrm{H}) ;{ }^{13} \mathrm{C}$ NMR $\left(100 \mathrm{MHz}, \mathrm{CDCl}_{3}\right) \delta: 177.4,142.9$, $137.4,137.2,133.8,128.5,128.1,128.0,127.3,126.6$, $126.4,125.2,122.5,121.7,121.5,119.2,114.5,109.8$, 108.1, 52.6, 49.9, 26.5; IR (KBr) v: 3451, 2928, 1701, $1627,740 \mathrm{~cm}^{-1}$; HRMS (ESI) calcd for $\mathrm{C}_{39} \mathrm{H}_{32} \mathrm{~N}_{3} \mathrm{O}[\mathrm{M}+$ $\mathrm{H}]^{+}$558.2540, found 558.2538.

(E)-3,3'-(1-(4-Bromophenyl)-3-phenylprop-2-ene-1,1diyl)bis(1-benzyl-1H-indole) (3x): White solid, $25.7 \mathrm{mg}$, $31 \%$ yield, reaction time 11 h. m.p. $131.6 \sim 132.1{ }^{\circ} \mathrm{C} ;{ }^{1} \mathrm{H}$ NMR (400 MHz, $\left.\mathrm{CDCl}_{3}\right) \delta: 7.38(\mathrm{dd}, J=8.0,8.0 \mathrm{~Hz}, 4 \mathrm{H})$, $7.33 \sim 7.14(\mathrm{~m}, 14 \mathrm{H}), 7.09(\mathrm{t}, J=8.0 \mathrm{~Hz}, 3 \mathrm{H}), 6.99(\mathrm{~d}, J=$ $8.0 \mathrm{~Hz}, 5 \mathrm{H}), 6.92(\mathrm{dt}, J=8.0,8.0 \mathrm{~Hz}, 2 \mathrm{H}), 6.64(\mathrm{~s}, 2 \mathrm{H})$, $5.93(\mathrm{~s}, 1 \mathrm{H}), 5.15(\mathrm{~m}, 4 \mathrm{H}) ;{ }^{13} \mathrm{C} \mathrm{NMR}\left(100 \mathrm{MHz}, \mathrm{CDCl}_{3}\right) \delta$ : $144.1,137.8,137.7,137.0,130.8,130.0,128.7,128.6$, $128.6,128.2,127.9,127.8,127.4,127.3,126.4,126.1$, $121.6,121.4,120.2,118.9,118.8,109.7,49.9,40.3$; IR (KBr) $v: 3452,1637,1399,733 \mathrm{~cm}^{-1}$; HRMS (ESI) calcd for $\mathrm{C}_{45} \mathrm{H}_{36} \mathrm{BrN}_{2}[\mathrm{M}+\mathrm{H}]^{+}$683.2056, found 683.2052.

3-(1-Benzyl-1H-indol-3-yl)-1-(4-bromophenyl)-3phenylpropan-1-one (4): White solid, $28.1 \mathrm{mg}, 47 \%$ yield, reaction time 11 h. m.p. 85.1 $\sim 85.4{ }^{\circ} \mathrm{C} ;{ }^{1} \mathrm{H}$ NMR $(400$ $\left.\mathrm{MHz}, \mathrm{CDCl}_{3}\right) \delta: 7.67(\mathrm{~d}, J=8.0 \mathrm{~Hz}, 2 \mathrm{H}), 7.44(\mathrm{dd}, J=8.0$, $8.0 \mathrm{~Hz}, 3 \mathrm{H}), 7.31(\mathrm{~d}, J=4.0 \mathrm{~Hz}, 2 \mathrm{H}), 7.22 \sim 7.08(\mathrm{~m}, 8 \mathrm{H})$, $6.98(\mathrm{~d}, J=4.0 \mathrm{~Hz}, 3 \mathrm{H}), 6.89(\mathrm{~s}, 1 \mathrm{H}), 5.16(\mathrm{~s}, 2 \mathrm{H}), 5.03(\mathrm{t}$, $J=4.0 \mathrm{~Hz}, 1 \mathrm{H}), 3.75 \sim 3.59(\mathrm{~m}, 2 \mathrm{H}) ;{ }^{13} \mathrm{C} \mathrm{NMR}(100 \mathrm{MHz}$, $\left.\mathrm{CDCl}_{3}\right) \delta: 197.5,144.0,137.5,136.9,135.8,131.7,129.5$, $128.6,128.4,128.0,127.7,127.4,127.2,126.5,126.3$, $125.5,121.9,119.6,119.1,118.2,109.7,49.8,45.0,38.3$; IR (KBr) v: 3451, 1637, 1398, $741 \mathrm{~cm}^{-1}$; HRMS (ESI) calcd for $\mathrm{C}_{30} \mathrm{H}_{25} \mathrm{BrNO}[\mathrm{M}+\mathrm{H}]^{+}$494.1114, found 494.1112.

\subsection{General procedure for the formation of} tri(indolyl)- methane 6

To a $5.0 \mathrm{~mL}$ vial were successively added 3-indolyl alcohol 5 (0.12 mmol), $N$-benzyl indole (2a) $(0.18 \mathrm{mmol})$, $\mathrm{CuBr}_{2}(5.4 \mathrm{mg}, 0.024 \mathrm{mmol})$ and $1.0 \mathrm{~mL}$ of $\mathrm{CH}_{3} \mathrm{CN}$. The resulting mixture was stirred at $35{ }^{\circ} \mathrm{C}$ for $20 \mathrm{~min}$ till almost full consumption of $\mathbf{5}$ monitored by TLC, and then the reaction mixture was directly subjected to flash column chromatography on silica gel $[V$ (petroleum ether) $: V$ (ethyl acetate) $=5: 1]$ to afford the corresponding product 6 as white solid in $54 \%$ yield.

1-Benzyl-1'-methyl-1H,1"H-[3,3':3',3"-terindol]-2'(1'H)one (6): White solid, $30.5 \mathrm{mg}, 54 \%$ yield, reaction time 20 
min. m.p. $142.5 \sim 143.1{ }^{\circ} \mathrm{C} ;{ }^{1} \mathrm{H}$ NMR (400 $\left.\mathrm{MHz}, \mathrm{CDCl}_{3}\right)$ $\delta: 8.14(\mathrm{~s}, 1 \mathrm{H}), 7.39(\mathrm{~d}, J=8.0 \mathrm{~Hz}, 1 \mathrm{H}), 7.32 \sim 7.28(\mathrm{~m}$, $3 \mathrm{H}), 7.25 \sim 7.15(\mathrm{~m}, 5 \mathrm{H}), 7.03(\mathrm{q}, J=8.0 \mathrm{~Hz}, 4 \mathrm{H}), 6.96(\mathrm{t}$, $J=8.0 \mathrm{~Hz}, 2 \mathrm{H}), 6.92(\mathrm{~s}, 1 \mathrm{H}), 6.87$ (q, $J=8.0 \mathrm{~Hz}, 2 \mathrm{H}), 6.82$ $(\mathrm{d}, J=4.0 \mathrm{~Hz}, 1 \mathrm{H}), 5.15(\mathrm{~s}, 2 \mathrm{H}), 3.31(\mathrm{~s}, 3 \mathrm{H}) ;{ }^{13} \mathrm{C} \mathrm{NMR}$ $\left(100 \mathrm{MHz}, \mathrm{CDCl}_{3}\right) \delta: 177.8,142.9,137.4,137.2,136.9$, $133.9,128.6,128.5,128.0,127.4,126.6,126.5,125.8$, $125.2,124.1,122.7,121.7,121.7,121.3,121.2,119.2$, $115.1,114.3,111.3,109.8,108.2,52.7,49.9,26.6$, one carbon missing in the aromatic region; IR (KBr) v: 3454 , 2079, 1637, $1399 \mathrm{~cm}^{-1}$; HRMS (ESI) calcd for $\mathrm{C}_{32} \mathrm{H}_{26} \mathrm{~N}_{3} \mathrm{O}$ $[\mathrm{M}+\mathrm{H}]^{+}$468.2070, found 468.2069.

辅助材料(Supporting Information) ${ }^{1} \mathrm{H}$ NMR and ${ }^{13} \mathrm{C}$ NMR spectra copies of $\mathbf{3 a} \sim \mathbf{3 x}, \mathbf{4}$ and $\mathbf{6}$. The Supporting Information is available free of charge via the Internet at http://sioc-journal.cn/.

\section{References}

[1] For selected reviews, see: (a) Somei, M.; Yamada, F. Nat. Prod. Rep. 2005, 22, 73.

(b) Cacchi, S.; Fabrizi, G. Chem. Rev. 2005, 105, 2873

(c) Chen, F. E.; Huang, J. Chem. Rev. 2005, 105, 4671.

(d) Humphrey, G. R.; Kuethe, J. T. Chem. Rev. 2006, 106, 2875

(e) Zhang, Y. C.; Jiang, F.; Shi, F. Acc. Chem. Res. 2020, 53, 425.

[2] For selected examples, see: (a) Jiang, F.; Chen, K. W.; Wu, P.; Zhang, Y. C.; Jiao, Y. C.; Shi, F. Angew. Chem., Int. Ed. 2019, 58, 15104.

(b) Sun, M.; Ma, C.; Zhou, S. J.; Lou, S. F.; Xiao, J.; Jiao, Y. C.; Shi, F. Angew. Chem., Int. Ed. 2019, 58, 8703.

(c) Wang, H. Q.; Xu, M. M.; Wan, Y.; Mao, Y. J.; Mei, G. J.; Shi, F. Adv. Synth. Catal. 2018, 360, 1850.

(d) Bai, G. X.; Dong, F. Y.; Xu, L. B.; Liu, Y. J.; Wang, L.; Li, S. S. Org. Lett. 2019, 21, 6225.

(e) Liu, X. L.; Zhou, G.; Gong, Y.; Yao, Z.; Zuo, X.; Zhang, W. H.; Zhou, Y. Org. Lett. 2019, 21, 2528.

(f) Xu, S. W.; Liu, X. W.; Zuo, X.; Zhou, G.; Gong, Y.; Liu, X. L.; Zhou, Y. Adv. Synth. Catal. 2019, 361, 5328.

(g) Zhu, Z. Q.; Yin, L.; Wang, Y.; Shen, Y.; Li, C.; Mei, G. J.; Shi, F. Org. Chem. Front. 2017, 4, 57.

(h) Wang, X.; Li, G. F.; Sun, K.; Zhang, B. Chin. J. Org. Chem. 2020, 40, 913 (in Chinese).

(王薪, 李国锋, 孙凯, 张冰, 有机化学, 2020, 40, 913.)

(i) Sun, K.; Li, Y. L.; Feng, R. R.; Mu, S. Q.; Wang, X.; Zhang, B. J. Org. Chem. 2020, 85, 1001.

(j) Wang, X.; Wang, Q. L.; Xue, Y. R.; Sun, K.; Wu, L. L.; Zhang, B. Chem. Commun. 2020, 56, 4436.

(k) Sun, K.; Li, G. F.; Li, Y. Y.; Yu, J.; Zhao, Q.; Zhang, Z. G.; Zhang, G. S. Adv. Synth. Catal. 2020, 362, 1947.
[3] (a) Giuseppe, B.; Ines, B.; Raffaele, R.; Jacques, L.; Gevenieve, B. J. Nat. Prod. 1995, 58, 1254.

(b) Chang, Y. C.; Riby, J.; Chang, G. H. F.; Peng, B. C.; Firestone, G.; Bjeldanes, L. F. Biochem. Pharmacol. 1999, 58, 825.

(c) Lee, C. H.; Yao, C. F.; Huang, S. M.; Ko, S. K.; Tan, Y. H.; Lee-Chen, G. J.; Wang, Y. C. Cancer 2008, 113, 815.

(d) Kamal, A.; Srikanth, Y. V. V.; Khan, M. N. A.; Shaik, T. B.; Ashraf, M. Bioorg. Med. Chem. Lett. 2010, 20, 5229.

[4] (a) Garbe, T. R.; Kobayashi, M.; Shimizu, M.; Takesue, N.; Ozawa, M.; Yukawa, H. J. Nat. Prod. 2000, 63, 596.

(b) Mason, M. R.; Barnard, T. S.; Segla, M. F.; Xie, B. H.; Kirschbaum, K. J. Chem. Crystallogr. 2003, 33, 531.

[5] (a) Zeng, X. F.; Ji, S. J.; Su, X. M. Chin. J. Chem. 2008, 26, 413.

(b) Chakrabarty, M.; Sarkar, S.; Linden, A.; Stein, B. K. Synth. Commun. 2004, 34, 1801.

(c) Zhang, Z. H.; Lin, J. Synth. Commun. 2007, 37, 209.

(d) Akgün, E.; Pindur, U.; Müller, J. J. Heterocycl. Chem. 1983, 20, 1303.

[6] Bergman, J. J. Heterocycl. Chem. 1971, 8, 329.

[7] Selič, L.; Stanovnik, B. Tetrahedron 2001, 57, 3159.

[8] (a) El Sayed, M. T.; Ahmed, K. M.; Mahmoud, K.; Hilgeroth, A. Eur. J. Med. Chem. 2015, 90, 845.

(b) Noland, W. E.; Kumar, H. V.; Flick, G. C.; Aapros, C. L.; Yoon, J. H.; Wilt, A. C.; Dehkordi, N.; Thao, S.; Schneered, A. K.; Gao, S. M.; Tritch, K. J. Tetrahedron 2017, 73, 3913.

(c) Chakrabarty, M.; Sarkar, S. Tetrahedron Lett. 2002, 43, 1351.

(d) Veisi, H.; Maleki, B.; Eshbala, F. H.; Veisi, H.; Masti, R.; Ashrafi, S. S.; Baghayeri, M. RSC Adv. 2014, 4, 30683. (e) Gu, D. G.; Ji, S. J. Chin. J. Chem. 2008, 26, 578.

[9] Xiang, J. C.; Wang, J. G.; Wang, M.; Meng, X. G.; Wu, A. X. Org. Biomol. Chem. 2015, 13, 4240.

[10] (a) El Sayed, M. T.; Mahmoud, K.; Hilgeroth, A.; Fakhr, I. M. I. J. Heterocycl. Chem. 2016, 53, 188.

(b) Khaksar, S.; Vahdat, S. M.; Gholizadeh, M.; Talesh, S. M. J. Fluorine Chem. 2012, 136, 8.

[11] Wang, W. B.; Zhu, Y. S.; Guo, S. Q.; Wang, Q. L.; Bu, Z. W. Org. Biomol. Chem. 2016, 14, 4420.

[12] (a) Zhu, Y. S.; Zhou, J.; Jin, S. J.; Dong, H. H.; Guo, J. M.; Bai, X. G.; Wang, Q. L.; Bu, Z. W. Chem. Commun. 2017, 53, 11201.

(b) Wang, W. B.; Bai, X. G.; Jin, S. J.; Guo, J. M.; Zhao, Y.; Miao, H. J.; Zhu, Y. S.; Wang, Q. L.; Bu, Z. W. Org. Lett. 2018, 20, 3451. (c) Guo, J. M.; Miao, H. J.; Zhao, Y.; Bai, X. G.; Zhu, Y. S.; Wang, Q. L.; Bu, Z. W. Chem. Commun. 2019, 55, 5207.

(d) Zhang, K.; Han, H. B.; Wang, L. L.; Zhang, Z. Y.; Wang, Q. L.; Zhang, W. J.; Bu, Z. W. Chem. Commun. 2019, 55, 13681.

(e) Wang, L. L.; Han, H. B.; Cui, Z. H.; Zhao, J. W.; Bu, Z. W.; Wang, Q. L. Org. Lett. 2020, 22, 873.

(f) Miao, H. J.; Wang, L. L.; Han, H. B.; Zhao, Y. D.; Wang, Q. L.; Bu, Z. W. Chem. Sci. 2020, 11, 1418.

(g) Bai, X. G.; Miao, H. J.; Zhao, Y.; Wang, Q. L.; Bu, Z. W. Org. Lett. 2020, 22, 5068 .

[13] Mo, L. P.; Ma, Z. C.; Zhang, Z. H. Synth. Commun. 2005, 35, 1997. 